Supporting information for

\title{
Calcium, strontium, barium and ytterbium complexes with cyclooctatetraenyl or cyclononatetraenyl ligands
}

Marc D. Walter, Gotthelf Wolmershäuser, and Helmut Sitzmann*

FB Chemie der Technischen Universität Kaiserslautern, Erwin-Schrödinger-Str. 54, D-67663

Kaiserslautern, Germany

E-mail: sitzmann@ chemie.uni-kl.de

\section{Contents:}

1. $\left[\left(\mathrm{C}_{5} \mathrm{H}\left(\mathrm{CHMe}_{2}\right)_{4}\right) \mathrm{Ca}\left(\mu, \eta^{8}: \eta^{8}-\mathrm{C}_{8} \mathrm{H}_{8}\right) \mathrm{Ca}\left(\mathrm{C}_{5} \mathrm{H}\left(\mathrm{CHMe}_{2}\right)_{4}\right)\right](\mathbf{2}-\mathrm{Ca}) \quad 2$

2. $\left[\left(\mathrm{C}_{5} \mathrm{H}\left(\mathrm{CHMe}_{2}\right)_{4}\right) \mathrm{Ba}\left(\mu, \eta^{8}: \eta^{8}-\mathrm{C}_{8} \mathrm{H}_{8}\right) \mathrm{Ba}\left(\mathrm{C}_{5} \mathrm{H}\left(\mathrm{CHMe}_{2}\right)_{4}\right)\right](2-\mathrm{Ba})$

3. Selected data collection and structure solution parameters (2-Ca, Ba) 14

4. SDD Basis Sets for Ba and Sr (Gaussian Input)

5. Cartesian Coordinates of Fully Optimized Structures 16

6. Complete list of authors for Gaussian 98 program for Ref. $80 \quad 20$ 


\section{1. $\left[\left(\mathrm{C}_{5} \mathrm{H}\left(\mathrm{CHMe}_{2}\right)_{4}\right) \mathrm{Ca}\left(\mu, \eta^{8}: \eta^{8}-\mathrm{C}_{8} \mathrm{H}_{8}\right) \mathrm{Ca}\left(\mathrm{C}_{5} \mathrm{H}\left(\mathrm{CHMe}_{2}\right)_{4}\right)\right] \quad$ (2-Ca)}

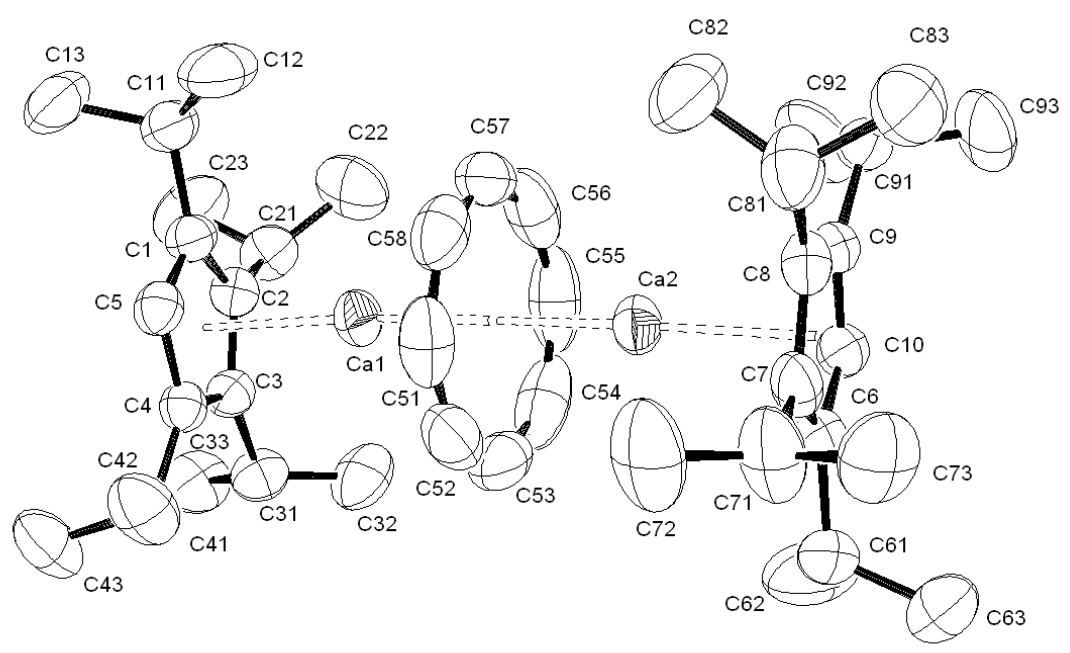

Figure S1. ORTEP diagram of $\left[\left(\mathrm{C}_{5} \mathrm{H}\left(\mathrm{CHMe}_{2}\right)_{4}\right) \mathrm{Ca}\left(\mu, \eta^{8}: \eta^{8}-\mathrm{C}_{8} \mathrm{H}_{8}\right) \mathrm{Ca}\left(\mathrm{C}_{5} \mathrm{H}\left(\mathrm{CHMe}_{2}\right)_{4}\right)\right] \quad$ (50\% probability ellipsoids).

1.1 Positional Parameters of $\left[\left(\mathrm{C}_{5} \mathrm{H}\left(\mathrm{CHMe}_{2}\right)_{4}\right) \mathrm{Ca}\left(\mu, \eta^{8}: \eta^{8}-\mathrm{C}_{8} \mathrm{H}_{8}\right) \mathrm{Ca}\left(\mathrm{C}_{5} \mathrm{H}\left(\mathrm{CHMe}_{2}\right)_{4}\right)\right]$ 1.1.1 Positional Parameters of Non-Hydrogen atoms of $\left[\left(\mathrm{C}_{5} \mathrm{H}\left(\mathrm{CHMe}_{2}\right)_{4}\right) \mathrm{Ca}\left(\mu, \eta^{8}: \eta^{8}-\mathrm{C}_{8} \mathrm{H}_{8}\right) \mathrm{Ca}\left(\mathrm{C}_{5} \mathrm{H}\left(\mathrm{CHMe}_{2}\right)_{4}\right)\right]$

\begin{tabular}{|c|c|c|c|c|}
\hline & $\mathrm{x}$ & $\mathrm{Y}$ & $z$ & $\mathrm{U}(\mathrm{eq})$ \\
\hline $\mathrm{Ca}(1)$ & $-1075(1)$ & $4108(1)$ & $2456(1)$ & $53(1)$ \\
\hline $\mathrm{Ca}(2)$ & $968(1)$ & $2844(1)$ & $2162(1)$ & $52(1)$ \\
\hline$C(1)$ & $-1313(3)$ & $5059(1)$ & $2843(2)$ & $49(1)$ \\
\hline$C(2)$ & $-2336(3)$ & $4987(1)$ & $2083(2)$ & $47(1)$ \\
\hline$C(3)$ & $-3398(3)$ & $4655(1)$ & $2272(2)$ & $46(1)$ \\
\hline$C(4)$ & $-3023(3)$ & $4527(1)$ & $3145(2)$ & $44(1)$ \\
\hline$C(5)$ & $-1743(3)$ & $4775(1)$ & $3484(2)$ & $48(1)$ \\
\hline$C(6)$ & $902(3)$ & $1879(1)$ & $1775(2)$ & $50(1)$ \\
\hline$C(7)$ & $1901(3)$ & $1922(1)$ & $2547(2)$ & $54(1)$ \\
\hline$C(8)$ & $3094(3)$ & $2203(1)$ & $2399(2)$ & $49(1)$ \\
\hline$C(9)$ & $2815(3)$ & $2333(1)$ & $1527(2)$ & $44(1)$ \\
\hline$C(10)$ & $1477(3)$ & $2134(1)$ & $1158(2)$ & $48(1)$ \\
\hline$C(11)$ & $-37(3)$ & $5407(1)$ & $3002(2)$ & 61 (1) \\
\hline$C(12)$ & $1343(3)$ & $5135(1)$ & $3331(3)$ & $108(2)$ \\
\hline$C(13)$ & $-204(4)$ & $5830(1)$ & $3605(2)$ & $86(1)$ \\
\hline$C(21)$ & $-2383(3)$ & $5246(1)$ & $1236(2)$ & $64(1)$ \\
\hline$C(22)$ & $-1135(4)$ & $5114(2)$ & $825(2)$ & $96(1)$ \\
\hline$C(23)$ & $-2591(5)$ & $5810(1)$ & $1293(3)$ & $100(1)$ \\
\hline$C(31)$ & $-4768(3)$ & $4490(1)$ & $1686(2)$ & $60(1)$ \\
\hline$C(32)$ & $-4582(4)$ & $4191(1)$ & $914(2)$ & $85(1)$ \\
\hline$C(33)$ & $-5822(3)$ & $4916(1)$ & $1455(2)$ & $83(1)$ \\
\hline$C(41)$ & $-3895(3)$ & $4225(1)$ & $3655(2)$ & $54(1)$ \\
\hline$C(42)$ & $-3006(4)$ & $3958(1)$ & $4413(2)$ & $77(1)$ \\
\hline$C(43)$ & $-4984(4)$ & $4561(1)$ & $3948(2)$ & $88(1)$ \\
\hline$C(51)$ & $244(5)$ & $3366(1)$ & $3441(2)$ & $77(1)$ \\
\hline$C(52)$ & $-985(5)$ & $3155(1)$ & $3017(3)$ & $84(1)$ \\
\hline$C(53)$ & $-1681(4)$ & $3126(1)$ & $2180(4)$ & $96(1)$ \\
\hline$C(54)$ & $-1408(5)$ & $3305(2)$ & $1401(3)$ & $105(2)$ \\
\hline$C(55)$ & $-308(7)$ & $3591(2)$ & $1178(2)$ & $105(2)$ \\
\hline$C(56)$ & $934(6)$ & $3805(1)$ & $1624(4)$ & $94(1)$ \\
\hline$C(57)$ & $1612(3)$ & $3821(1)$ & $2474(3)$ & $82(1)$ \\
\hline$C(58)$ & $1315(4)$ & $3643(1)$ & $3222(2)$ & $76(1)$ \\
\hline$C(61)$ & $-478(3)$ & $1584(1)$ & $1607(2)$ & $68(1)$ \\
\hline$C(62)$ & $-1543(4)$ & $1780(2)$ & $811(3)$ & $117(2)$ \\
\hline
\end{tabular}




\begin{tabular}{rrrrr}
$C(63)$ & $-222(4)$ & $1030(1)$ & $1478(2)$ & $84(1)$ \\
$C(71)$ & $1732(4)$ & $1666(1)$ & $3372(2)$ & $84(1)$ \\
$C(72)$ & $1432(5)$ & $1980(2)$ & $4046(2)$ & $105(1)$ \\
$C(73)$ & $2665(4)$ & $1216(1)$ & $3614(2)$ & $83(1)$ \\
$C(81)$ & $4430(3)$ & $2313(1)$ & $3048(2)$ & $75(1)$ \\
$C(82)$ & $4657(4)$ & $2837(1)$ & $3322(3)$ & $103(1)$ \\
$C(83)$ & $5706(3)$ & $2026(1)$ & $2929(3)$ & $96(1)$ \\
$C(91)$ & $3790(3)$ & $2587(1)$ & $1022(2)$ & $56(1)$ \\
$C(92)$ & $3051(4)$ & $2974(2)$ & $408(3)$ & $120(2)$ \\
$C(93)$ & $4499(4)$ & $2211(1)$ & $553(3)$ & $101(1)$ \\
\hline
\end{tabular}

1.1.2 Positional Parameters of Hydrogen atoms of $\left[\left(\mathrm{C}_{5} \mathrm{H}\left(\mathrm{CHMe}_{2}\right)_{4}\right) \mathrm{Ca}\left(\mu, \eta^{8}: \eta^{8}-\mathrm{C}_{8} \mathrm{H}_{8}\right) \mathrm{Ca}\left(\mathrm{C}_{5} \mathrm{H}\left(\mathrm{CHMe}_{2}\right)_{4}\right)\right]$

\begin{tabular}{|c|c|c|c|c|}
\hline & $x$ & $\mathrm{Y}$ & $z$ & $\mathrm{U}(\mathrm{eq})$ \\
\hline $\mathrm{H}(5)$ & -1256 & 4755 & 4046 & 57 \\
\hline $\mathrm{H}(10)$ & 1038 & 2166 & 588 & 57 \\
\hline $\mathrm{H}(11)$ & 30 & 5559 & 2456 & 73 \\
\hline $\mathrm{H}(12 \mathrm{~A})$ & 2111 & 5372 & 3429 & 161 \\
\hline $\mathrm{H}(12 \mathrm{~B})$ & 1501 & 4891 & 2918 & 161 \\
\hline $\mathrm{H}(12 \mathrm{C})$ & 1294 & 4967 & 3854 & 161 \\
\hline $\mathrm{H}(13 \mathrm{~A})$ & -278 & 5693 & 4148 & 129 \\
\hline $\mathrm{H}(13 \mathrm{~B})$ & -1048 & 6018 & 3375 & 129 \\
\hline $\mathrm{H}(13 \mathrm{C})$ & 608 & 6048 & 3673 & 129 \\
\hline $\mathrm{H}(21)$ & -3235 & 5119 & 851 & 77 \\
\hline $\mathrm{H}(22 \mathrm{~A})$ & -264 & 5216 & 1193 & 144 \\
\hline $\mathrm{H}(22 \mathrm{~B})$ & -1235 & 5285 & 289 & 144 \\
\hline $\mathrm{H}(22 \mathrm{C})$ & -1118 & 4757 & 734 & 144 \\
\hline $\mathrm{H}(23 \mathrm{~A})$ & -3422 & 5876 & 1523 & 150 \\
\hline $\mathrm{H}(23 \mathrm{~B})$ & -2709 & 5956 & 736 & 150 \\
\hline $\mathrm{H}(23 \mathrm{C})$ & -1774 & 5956 & 1658 & 150 \\
\hline $\mathrm{H}(31)$ & -5216 & 4257 & 2026 & 72 \\
\hline $\mathrm{H}(32 \mathrm{~A})$ & -5498 & 4101 & 587 & 128 \\
\hline $\mathrm{H}(32 \mathrm{~B})$ & -4045 & 3892 & 1095 & 128 \\
\hline $\mathrm{H}(32 \mathrm{C})$ & -4083 & 4392 & 571 & 128 \\
\hline $\mathrm{H}(33 \mathrm{~A})$ & -5454 & 5156 & 1109 & 124 \\
\hline $\mathrm{H}(33 \mathrm{~B})$ & -5973 & 5076 & 1966 & 124 \\
\hline $\mathrm{H}(33 \mathrm{C})$ & -6708 & 4784 & 1145 & 124 \\
\hline $\mathrm{H}(41)$ & -4416 & 3969 & 3277 & 65 \\
\hline $\mathrm{H}(42 \mathrm{~A})$ & -2336 & 3740 & 4223 & 115 \\
\hline $\mathrm{H}(42 \mathrm{~B})$ & -3615 & 3762 & 4694 & 115 \\
\hline $\mathrm{H}(42 \mathrm{C})$ & -2504 & 4201 & 4803 & 115 \\
\hline $\mathrm{H}(43 \mathrm{~A})$ & -4502 & 4812 & 4330 & 132 \\
\hline $\mathrm{H}(43 \mathrm{~B})$ & -5573 & 4361 & 4236 & 132 \\
\hline $\mathrm{H}(43 \mathrm{C})$ & -5565 & 4721 & 3463 & 132 \\
\hline $\mathrm{H}(51)$ & 392 & 3309 & 4025 & 93 \\
\hline $\mathrm{H}(52)$ & -1473 & 2989 & 3381 & 101 \\
\hline $\mathrm{H}(53)$ & -2529 & 2947 & 2112 & 115 \\
\hline $\mathrm{H}(54)$ & -2106 & 3214 & 934 & 126 \\
\hline $\mathrm{H}(55)$ & -449 & 3651 & 595 & 126 \\
\hline $\mathrm{H}(56)$ & 1431 & 3978 & 1273 & 113 \\
\hline $\mathrm{H}(57)$ & 2470 & 3994 & 2557 & 99 \\
\hline $\mathrm{H}(58)$ & 2010 & 3731 & 3692 & 91 \\
\hline $\mathrm{H}(61)$ & -921 & 1620 & 2103 & 82 \\
\hline $\mathrm{H}(62 \mathrm{~A})$ & -1104 & 1768 & 324 & 175 \\
\hline $\mathrm{H}(62 \mathrm{~B})$ & -1807 & 2120 & 907 & 175 \\
\hline $\mathrm{H}(62 \mathrm{C})$ & -2377 & 1571 & 710 & 175 \\
\hline $\mathrm{H}(63 \mathrm{~A})$ & 163 & 986 & 975 & 126 \\
\hline $\mathrm{H}(63 \mathrm{~B})$ & -1105 & 851 & 1416 & 126 \\
\hline $\mathrm{H}(63 \mathrm{C})$ & 438 & 902 & 1963 & 126 \\
\hline $\mathrm{H}(71)$ & 808 & 1502 & 3185 & 101 \\
\hline $\mathrm{H}(72 \mathrm{~A})$ & 1263 & 1773 & 4505 & 157 \\
\hline $\mathrm{H}(72 \mathrm{~B})$ & 605 & 2180 & 3834 & 157 \\
\hline $\mathrm{H}(72 \mathrm{C})$ & 2230 & 2196 & 4247 & 157 \\
\hline $\mathrm{H}(73 \mathrm{~A})$ & 3626 & 1324 & 3822 & 124 \\
\hline $\mathrm{H}(73 \mathrm{~B})$ & 2628 & 1005 & 3124 & 124 \\
\hline
\end{tabular}




\begin{tabular}{lrrrr}
$H(73 C)$ & 2338 & 1030 & 4051 & 124 \\
$H(81)$ & 4219 & 2154 & 3559 & 90 \\
$H(82 A)$ & 5489 & 2859 & 3768 & 155 \\
$H(82 B)$ & 3845 & 2955 & 3529 & 155 \\
$H(82 C)$ & 4787 & 3040 & 2848 & 155 \\
$H(83 A)$ & 6032 & 2155 & 2443 & 144 \\
$H(83 B)$ & 5465 & 1677 & 2841 & 144 \\
$H(83 C)$ & 6446 & 2062 & 3427 & 144 \\
$H(91)$ & 4537 & 2760 & 1428 & 67 \\
$H(92 A)$ & 3722 & 3125 & 115 & 181 \\
$H(92 B)$ & 2650 & 3228 & 714 & 181 \\
$H(92 C)$ & 2307 & 2816 & 3 & 181 \\
$H(93 A)$ & 3798 & 2051 & 122 & 151 \\
$H(93 B)$ & 4968 & 1962 & 945 & 151 \\
$H(93 C)$ & 5186 & 2378 & 290 & 151 \\
\hline
\end{tabular}

1.2 Thermal Parameters of $\left[\left(\mathrm{C}_{5} \mathrm{H}\left(\mathrm{CHMe}_{2}\right)_{4}\right) \mathrm{Ca}\left(\mu, \eta^{8}: \eta^{8}-\mathrm{C}_{8} \mathrm{H}_{8}\right) \mathrm{Ca}\left(\mathrm{C}_{5} \mathrm{H}\left(\mathrm{CHMe}_{2}\right)_{4}\right)\right]$

\begin{tabular}{|c|c|c|c|c|c|c|}
\hline & $\mathrm{U}_{11}$ & $\mathrm{U}_{22}$ & $\mathrm{U}_{33}$ & $\mathrm{U}_{23}$ & $\mathrm{U}_{13}$ & $\mathrm{U}_{12}$ \\
\hline $\mathrm{Ca}(1)$ & $51(1)$ & $48(1)$ & $60(1)$ & $-3(1)$ & $14(1)$ & $10(1)$ \\
\hline $\mathrm{Ca}(2)$ & $56(1)$ & $45(1)$ & $58(1)$ & $-5(1)$ & $18(1)$ & $7(1)$ \\
\hline$C(1)$ & $43(1)$ & $46(1)$ & $58(2)$ & $-5(1)$ & $8(1)$ & $-3(1)$ \\
\hline$C(2)$ & $47(1)$ & $48(1)$ & $44(2)$ & $1(1)$ & $6(1)$ & $3(1)$ \\
\hline$C(3)$ & $40(1)$ & $44(1)$ & $52(2)$ & $-3(1)$ & $4(1)$ & $2(1)$ \\
\hline$C(4)$ & $43(1)$ & $42(1)$ & $45(2)$ & $-1(1)$ & $8(1)$ & $4(1)$ \\
\hline$C(5)$ & $48(1)$ & $50(1)$ & $43(2)$ & $-5(1)$ & $4(1)$ & $2(1)$ \\
\hline$C(6)$ & $55(2)$ & $44(1)$ & $56(2)$ & $-6(1)$ & $24(1)$ & $-4(1)$ \\
\hline$C(7)$ & $71(2)$ & $44(1)$ & $57(2)$ & $6(1)$ & $35(2)$ & $9(1)$ \\
\hline$C(8)$ & $53(2)$ & $47(1)$ & $46(2)$ & $-6(1)$ & $10(1)$ & $10(1)$ \\
\hline$C(9)$ & $47(1)$ & $44(1)$ & $45(2)$ & $-7(1)$ & $19(1)$ & $-3(1)$ \\
\hline$C(10)$ & $54(2)$ & $48(1)$ & $44(2)$ & $-4(1)$ & $17(1)$ & $-4(1)$ \\
\hline$C(11)$ & $55(2)$ & $54(2)$ & $73(2)$ & $-7(1)$ & $10(2)$ & $-9(1)$ \\
\hline$C(12)$ & $47(2)$ & $72(2)$ & $198(5)$ & $-31(3)$ & $12(2)$ & $-7(2)$ \\
\hline$C(13)$ & $79(2)$ & $59(2)$ & $114(3)$ & $-29(2)$ & $4(2)$ & $-11(2)$ \\
\hline$C(21)$ & $74(2)$ & $60(2)$ & $56(2)$ & $10(1)$ & $8(2)$ & $-4(1)$ \\
\hline$C(22)$ & $98(3)$ & $128(3)$ & $69(2)$ & $19(2)$ & $32(2)$ & $-10(2)$ \\
\hline$C(23)$ & $140(4)$ & $56(2)$ & $92(3)$ & $24(2)$ & $-6(2)$ & $-9(2)$ \\
\hline$C(31)$ & $50(2)$ & $64(2)$ & $60(2)$ & $2(1)$ & $-1(1)$ & $-2(1)$ \\
\hline$C(32)$ & $81(2)$ & $82(2)$ & $81(3)$ & $-20(2)$ & $-13(2)$ & $-5(2)$ \\
\hline C (33) & $56(2)$ & $87(2)$ & $97(3)$ & $6(2)$ & $-6(2)$ & $13(2)$ \\
\hline$C(41)$ & $55(2)$ & $56(2)$ & $54(2)$ & $-3(1)$ & $14(1)$ & $-6(1)$ \\
\hline$C(42)$ & $88(2)$ & $80(2)$ & $71(2)$ & $22(2)$ & $34(2)$ & $10(2)$ \\
\hline$C(43)$ & $75(2)$ & $95(2)$ & $106(3)$ & $15(2)$ & $51(2)$ & $20(2)$ \\
\hline$C(51)$ & $118(3)$ & $65(2)$ & $52(2)$ & $6(2)$ & $27(2)$ & $33(2)$ \\
\hline$C(52)$ & $96(3)$ & $59(2)$ & $117(4)$ & $6(2)$ & $67(3)$ & $5(2)$ \\
\hline$C(53)$ & $51(2)$ & $64(2)$ & $172(5)$ & $-36(3)$ & $22(3)$ & $-7(2)$ \\
\hline$C(54)$ & $93(3)$ & $95(3)$ & $101(4)$ & $-49(3)$ & $-41(3)$ & $43(3)$ \\
\hline$C(55)$ & $170(5)$ & $95(3)$ & $49(2)$ & $7(2)$ & $21(3)$ & $72(3)$ \\
\hline$C(56)$ & $133(4)$ & $60(2)$ & $116(4)$ & $27(2)$ & $87(3)$ & $27(2)$ \\
\hline$C(57)$ & $57(2)$ & $47(2)$ & $151(4)$ & $-13(2)$ & $41(2)$ & $-2(1)$ \\
\hline$C(58)$ & $64(2)$ & $65(2)$ & $89(3)$ & $-21(2)$ & $-6(2)$ & $18(2)$ \\
\hline$C(61)$ & $71(2)$ & $61(2)$ & $82(2)$ & $-12(2)$ & $36(2)$ & $-17(2)$ \\
\hline$C(62)$ & $66(2)$ & $102(3)$ & $167(4)$ & $18(3)$ & $-12(3)$ & $-21(2)$ \\
\hline$C(63)$ & $86(2)$ & $61(2)$ & $105(3)$ & $-14(2)$ & $17(2)$ & $-14(2)$ \\
\hline$C(71)$ & $128(3)$ & $72(2)$ & $66(2)$ & $22(2)$ & $50(2)$ & $31(2)$ \\
\hline$C(72)$ & $161(4)$ & $105(3)$ & $61(2)$ & $16(2)$ & $53(3)$ & $38(3)$ \\
\hline$C(73)$ & $108(3)$ & $63(2)$ & $79(2)$ & $18(2)$ & $19(2)$ & $11(2)$ \\
\hline$C(81)$ & $68(2)$ & $79(2)$ & $70(2)$ & $-18(2)$ & $-3(2)$ & $22(2)$ \\
\hline$C(82)$ & $73(2)$ & $69(2)$ & $151(4)$ & $-36(2)$ & $-16(2)$ & $1(2)$ \\
\hline$C(83)$ & $58(2)$ & $83(2)$ & $138(4)$ & $-17(2)$ & $-1(2)$ & $18(2)$ \\
\hline$C(91)$ & $59(2)$ & $54(2)$ & $59(2)$ & $-9(1)$ & $24(1)$ & $-12(1)$ \\
\hline$C(92)$ & $125(3)$ & $99(3)$ & $166(4)$ & $64(3)$ & $98(3)$ & $32(3)$ \\
\hline$C(93)$ & $129(3)$ & $80(2)$ & $124(3)$ & $8(2)$ & $98(3)$ & $12(2)$ \\
\hline
\end{tabular}


1.3 Bond distances $(\AA)$ for $\left[\left(\mathrm{C}_{5} \mathrm{H}\left(\mathrm{CHMe}_{2}\right)_{4}\right) \mathrm{Ca}\left(\mu, \eta^{8}: \eta^{8}-\mathrm{C}_{8} \mathrm{H}_{8}\right) \mathrm{Ca}\left(\mathrm{C}_{5} \mathrm{H}\left(\mathrm{CHMe}_{2}\right)_{4}\right)\right]$

\begin{tabular}{|c|c|c|c|}
\hline $\mathrm{Ca}(1)-\mathrm{C}(5)$ & $2.589(2)$ & $C(6)-C(10)$ & $1.401(3)$ \\
\hline $\mathrm{Ca}(1)-\mathrm{C}(4)$ & $2.606(2)$ & $C(6)-C(7)$ & $1.410(4)$ \\
\hline $\mathrm{Ca}(1)-\mathrm{C}(1)$ & $2.626(3)$ & $C(6)-C(61)$ & $1.517(4)$ \\
\hline $\mathrm{Ca}(1)-\mathrm{C}(3)$ & $2.629(2)$ & $C(7)-C(8)$ & $1.427(4)$ \\
\hline $\mathrm{Ca}(1)-\mathrm{C}(2)$ & $2.645(2)$ & $C(7)-C(71)$ & $1.525(4)$ \\
\hline $\mathrm{Ca}(1)-\mathrm{C}(58)$ & $2.677(3)$ & $C(8)-C(9)$ & $1.414(3)$ \\
\hline $\mathrm{Ca}(1)-\mathrm{C}(51)$ & $2.680(3)$ & $C(8)-C(81)$ & $1.508(4)$ \\
\hline $\mathrm{Ca}(1)-\mathrm{C}(56)$ & $2.682(3)$ & $C(9)-C(10)$ & $1.405(3)$ \\
\hline $\mathrm{Ca}(1)-\mathrm{C}(57)$ & $2.684(3)$ & $C(9)-C(91)$ & $1.516(3)$ \\
\hline $\mathrm{Ca}(1)-\mathrm{C}(52)$ & $2.686(3)$ & $C(11)-C(12)$ & $1.508(4)$ \\
\hline $\mathrm{Ca}(1)-\mathrm{C}(53)$ & $2.692(3)$ & $C(11)-C(13)$ & $1.512(4)$ \\
\hline $\mathrm{Ca}(1)-\mathrm{C}(55)$ & $2.692(4)$ & $C(21)-C(22)$ & $1.521(4)$ \\
\hline $\mathrm{Ca}(2)-\mathrm{C}(10)$ & $2.592(2)$ & $C(21)-C(23)$ & $1.518(4)$ \\
\hline $\mathrm{Ca}(2)-\mathrm{C}(9)$ & $2.600(2)$ & $C(31)-C(32)$ & $1.511(4)$ \\
\hline $\mathrm{Ca}(2)-\mathrm{C}(8)$ & $2.627(2)$ & $C(31)-C(33)$ & $1.514(4)$ \\
\hline $\mathrm{Ca}(2)-\mathrm{C}(6)$ & $2.637(2)$ & $C(41)-C(42)$ & $1.515(4)$ \\
\hline $\mathrm{Ca}(2)-\mathrm{C}(7)$ & $2.640(3)$ & $C(41)-C(43)$ & $1.519(4)$ \\
\hline $\mathrm{Ca}(2)-\mathrm{C}(53)$ & $2.658(3)$ & $C(51)-C(52)$ & $1.359(5)$ \\
\hline $\mathrm{Ca}(2)-\mathrm{C}(54)$ & $2.660(4)$ & $C(51)-C(58)$ & $1.367(5)$ \\
\hline $\mathrm{Ca}(2)-\mathrm{C}(52)$ & $2.669(3)$ & $C(52)-C(53)$ & $1.375(6)$ \\
\hline $\mathrm{Ca}(2)-\mathrm{C}(55)$ & $2.677(4)$ & $C(53)-C(54)$ & $1.411(6)$ \\
\hline $\mathrm{Ca}(2)-\mathrm{C}(51)$ & $2.684(3)$ & $C(54)-C(55)$ & $1.405(7)$ \\
\hline $\mathrm{Ca}(2)-\mathrm{C}(56)$ & $2.696(3)$ & $C(55)-C(56)$ & $1.384(6)$ \\
\hline $\mathrm{Ca}(2)-\mathrm{C}(57)$ & 2.695 (3) & $C(56)-C(57)$ & $1.387(6)$ \\
\hline$C(1)-C(5)$ & $1.403(4)$ & $C(57)-C(58)$ & $1.373(5)$ \\
\hline$C(1)-C(2)$ & $1.416(4)$ & $C(61)-C(63)$ & $1.515(4)$ \\
\hline$C(1)-C(11)$ & $1.515(3)$ & $C(61)-C(62)$ & 1.555 (5) \\
\hline$C(2)-C(3)$ & $1.426(3)$ & $C(71)-C(72)$ & $1.440(4)$ \\
\hline$C(2)-C(21)$ & $1.517(4)$ & $C(71)-C(73)$ & $1.498(4)$ \\
\hline$C(3)-C(4)$ & $1.416(3)$ & $C(81)-C(82)$ & $1.464(4)$ \\
\hline$C(3)-C(31)$ & $1.518(4)$ & $C(81)-C(83)$ & $1.487(4)$ \\
\hline$C(4)-C(5)$ & $1.404(3)$ & $C(91)-C(93)$ & $1.497(4)$ \\
\hline$C(4)-C(41)$ & $1.514(3)$ & $C(91)-C(92)$ & $1.498(5)$ \\
\hline
\end{tabular}

1.4 Bond angles $\left(^{\circ}\right)$ for $\left[\left(\mathrm{C}_{5} \mathrm{H}\left(\mathrm{CHMe}_{2}\right)_{4}\right) \mathrm{Ca}\left(\mu, \eta^{8}: \eta^{8}-\mathrm{C}_{8} \mathrm{H}_{8}\right) \mathrm{Ca}\left(\mathrm{C}_{5} \mathrm{H}\left(\mathrm{CHMe}_{2}\right)_{4}\right)\right]$

\begin{tabular}{lllr}
\hline & & & \\
$\mathrm{C}(5)-\mathrm{Ca}(1)-\mathrm{C}(4)$ & $31.36(8)$ & $\mathrm{C}(58)-\mathrm{Ca}(1)-\mathrm{C}(51)$ & $29.57(11)$ \\
$\mathrm{C}(5)-\mathrm{Ca}(1)-\mathrm{C}(1)$ & $31.21(8)$ & $\mathrm{C}(5)-\mathrm{Ca}(1)-\mathrm{C}(56)$ & $144.92(14)$ \\
$\mathrm{C}(4)-\mathrm{Ca}(1)-\mathrm{C}(1)$ & $51.98(7)$ & $\mathrm{C}(4)-\mathrm{Ca}(1)-\mathrm{C}(56)$ & $171.57(9)$ \\
$\mathrm{C}(5)-\mathrm{Ca}(1)-\mathrm{C}(3)$ & $51.58(8)$ & $\mathrm{C}(1)-\mathrm{Ca}(1)-\mathrm{C}(56)$ & $120.84(10)$ \\
$\mathrm{C}(4)-\mathrm{Ca}(1)-\mathrm{C}(3)$ & $31.37(8)$ & $\mathrm{C}(3)-\mathrm{Ca}(1)-\mathrm{C}(56)$ & $142.13(13)$ \\
$\mathrm{C}(1)-\mathrm{Ca}(1)-\mathrm{C}(3)$ & $51.75(8)$ & $\mathrm{C}(2)-\mathrm{Ca}(1)-\mathrm{C}(56)$ & $119.69(9)$ \\
$\mathrm{C}(5)-\mathrm{Ca}(1)-\mathrm{C}(2)$ & $51.49(8)$ & $\mathrm{C}(51)-\mathrm{Ca}(1)-\mathrm{C}(56)$ & $76.85(14)$ \\
$\mathrm{C}(4)-\mathrm{Ca}(1)-\mathrm{C}(2)$ & $51.93(7)$ & $\mathrm{C}(5)-\mathrm{Ca}(1)-\mathrm{C}(57)$ & $123.31(11)$ \\
$\mathrm{C}(1)-\mathrm{Ca}(1)-\mathrm{C}(2)$ & $31.15(8)$ & $\mathrm{C}(4)-\mathrm{Ca}(1)-\mathrm{C}(57)$ & $152.52(12)$ \\
$\mathrm{C}(3)-\mathrm{Ca}(1)-\mathrm{C}(2)$ & $31.37(7)$ & $\mathrm{C}(1)-\mathrm{Ca}(1)-\mathrm{C}(57)$ & $113.61(9)$ \\
$\mathrm{C}(5)-\mathrm{Ca}(1)-\mathrm{C}(58)$ & $108.95(9)$ & $\mathrm{C}(3)-\mathrm{Ca}(1)-\mathrm{C}(57)$ & $161.77(9)$ \\
$\mathrm{C}(4)-\mathrm{Ca}(1)-\mathrm{C}(58)$ & $128.69(10)$ & $\mathrm{C}(58)-\mathrm{Ca}(1)-\mathrm{C}(57)$ & $130.42(9)$ \\
$\mathrm{C}(1)-\mathrm{Ca}(1)-\mathrm{C}(58)$ & $116.40(9)$ & $\mathrm{C}(51)-\mathrm{Ca}(1)-\mathrm{C}(57)$ & $29.67(12)$ \\
$\mathrm{C}(3)-\mathrm{Ca}(1)-\mathrm{C}(58)$ & $159.48(10)$ & $\mathrm{C}(56)-\mathrm{Ca}(1)-\mathrm{C}(57)$ & $56.38(13)$ \\
$\mathrm{C}(2)-\mathrm{Ca}(1)-\mathrm{C}(58)$ & $145.40(10)$ & $\mathrm{C}(5)-\mathrm{Ca}(1)-\mathrm{C}(52)$ & $29.96(13)$ \\
$\mathrm{C}(5)-\mathrm{Ca}(1)-\mathrm{C}(51)$ & $106.11(9)$ & $\mathrm{C}(1)-\mathrm{Ca}(1)-\mathrm{C}(52)$ & $115.40(10)$ \\
$\mathrm{C}(4)-\mathrm{Ca}(1)-\mathrm{C}(51)$ & $111.01(9)$ & $\mathrm{C}(3)-\mathrm{Ca}(1)-\mathrm{C}(52)$ & $103.80(8)$ \\
$\mathrm{C}(1)-\mathrm{Ca}(1)-\mathrm{C}(51)$ & $128.55(10)$ & & $145.97(11)$ \\
$\mathrm{C}(3)-\mathrm{Ca}(1)-\mathrm{C}(51)$ & $139.70(10)$ & & $121.95(10)$ \\
$\mathrm{C}(2)-\mathrm{Ca}(1)-\mathrm{C}(51)$ & $157.53(9)$ &
\end{tabular}


$\mathrm{C}(55)-\mathrm{Ca}(2)-\mathrm{C}(51)$

$\mathrm{C}(10)-\mathrm{Ca}(2)-\mathrm{C}(56)$

$\mathrm{C}(9)-\mathrm{Ca}(2)-\mathrm{C}(56)$

$\mathrm{C}(8)-\mathrm{Ca}(2)-\mathrm{C}(56)$

$\mathrm{C}(6)-\mathrm{Ca}(2)-\mathrm{C}(56)$

$\mathrm{C}(7)-\mathrm{Ca}(2)-\mathrm{C}(56)$

$\mathrm{C}(53)-\mathrm{Ca}(2)-\mathrm{C}(56)$

$\mathrm{C}(54)-\mathrm{Ca}(2)-\mathrm{C}(56)$

$\mathrm{C}(52)-\mathrm{Ca}(2)-\mathrm{C}(56)$

$\mathrm{C}(55)-\mathrm{Ca}(2)-\mathrm{C}(56)$

$\mathrm{C}(51)-\mathrm{Ca}(2)-\mathrm{C}(56)$

$\mathrm{C}(10)-\mathrm{Ca}(2)-\mathrm{C}(57)$

$\mathrm{C}(9)-\mathrm{Ca}(2)-\mathrm{C}(57)$

$\mathrm{C}(8)-\mathrm{Ca}(2)-\mathrm{C}(57)$

$\mathrm{C}(6)-\mathrm{Ca}(2)-\mathrm{C}(57)$

$\mathrm{C}(7)-\mathrm{Ca}(2)-\mathrm{C}(57)$

$\mathrm{C}(53)-\mathrm{Ca}(2)-\mathrm{C}(57)$

$\mathrm{C}(54)-\mathrm{Ca}(2)-\mathrm{C}(57)$

$\mathrm{C}(52)-\mathrm{Ca}(2)-\mathrm{C}(57)$

$\mathrm{C}(55)-\mathrm{Ca}(2)-\mathrm{C}(57)$

$\mathrm{C}(51)-\mathrm{Ca}(2)-\mathrm{C}(57)$

$\mathrm{C}(56)-\mathrm{Ca}(2)-\mathrm{C}(57)$

$C(5)-C(1)-C(2)$

$C(5)-C(1)-C(11)$

$C(2)-C(1)-C(11)$

$\mathrm{C}(5)-\mathrm{C}(1)-\mathrm{Ca}(1)$

$\mathrm{C}(2)-\mathrm{C}(1)-\mathrm{Ca}(1)$

$\mathrm{C}(11)-\mathrm{C}(1)-\mathrm{Ca}(1)$

$\mathrm{C}(1)-\mathrm{C}(2)-\mathrm{C}(3)$

$C(1)-C(2)-C(21)$

$C(3)-C(2)-C(21)$

$\mathrm{C}(1)-\mathrm{C}(2)-\mathrm{Ca}(1)$

$\mathrm{C}(3)-\mathrm{C}(2)-\mathrm{Ca}$ (1)

$\mathrm{C}(21)-\mathrm{C}(2)-\mathrm{Ca}(1)$

$C(4)-C(3)-C(2)$

$C(4)-C(3)-C(31)$

$\mathrm{C}(2)-\mathrm{C}(3)-\mathrm{C}(31)$

$\mathrm{C}(4)-\mathrm{C}(3)-\mathrm{Ca}(1)$

$\mathrm{C}(2)-\mathrm{C}(3)-\mathrm{Ca}(1)$

$\mathrm{C}(31)-\mathrm{C}(3)-\mathrm{Ca}(1)$

$C(5)-C(4)-C(3)$

$C(5)-C(4)-C(41)$

$\mathrm{C}(3)-\mathrm{C}(4)-\mathrm{C}(41)$

$\mathrm{C}(5)-\mathrm{C}(4)-\mathrm{Ca}(1)$

$\mathrm{C}(3)-\mathrm{C}(4)-\mathrm{Ca}(1)$

$\mathrm{C}(41)-\mathrm{C}(4)-\mathrm{Ca}(1)$

$\mathrm{C}(1)-\mathrm{C}(5)-\mathrm{C}(4)$

$\mathrm{C}(1)-\mathrm{C}(5)-\mathrm{Ca}(1)$

$\mathrm{C}(4)-\mathrm{C}(5)-\mathrm{Ca}(1)$

$C(10)-C(6)-C(7)$

$\mathrm{C}(10)-\mathrm{C}(6)-\mathrm{C}(61)$

$\mathrm{C}(7)-\mathrm{C}(6)-\mathrm{C}(61)$

$\mathrm{C}(10)-\mathrm{C}(6)-\mathrm{Ca}(2)$

$\mathrm{C}(7)-\mathrm{C}(6)-\mathrm{Ca}(2)$

$\mathrm{C}(61)-\mathrm{C}(6)-\mathrm{Ca}(2)$

$C(6)-C(7)-C(8)$

$C(6)-C(7)-C(71)$

$\mathrm{C}(8)-\mathrm{C}(7)-\mathrm{C}(71)$

$\mathrm{C}(6)-\mathrm{C}(7)-\mathrm{Ca}(2)$

$\mathrm{C}(8)-\mathrm{C}(7)-\mathrm{Ca}(2)$

$\mathrm{C}(71)-\mathrm{C}(7)-\mathrm{Ca}(2)$

$C(9)-C(8)-C(7)$

$C(9)-C(8)-C(81)$

$\mathrm{C}(7)-\mathrm{C}(8)-\mathrm{C}(81)$

$\mathrm{C}(9)-\mathrm{C}(8)-\mathrm{Ca}(2)$

$\mathrm{C}(7)-\mathrm{C}(8)-\mathrm{Ca}(2)$

$\mathrm{C}(81)-\mathrm{C}(8)-\mathrm{Ca}(2)$

$C(10)-C(9)-C(8)$

$C(10)-C(9)-C(91)$

$\mathrm{C}(8)-\mathrm{C}(9)-\mathrm{C}(91)$
$84.71(10)$

$118.93(11)$

$109.59(9)$

$128.34(11)$

$148.09(13)$

$159.55(10)$

$77.65(13)$

$57.46(16)$

84.64 (10)

$29.85(14)$

76.44 (11)

138.87 (10)

115.34 (9)

$117.17(9)$

167.32 (8)

142.94 (12)

$84.81(10)$

77.50 (13)

$76.38(11)$

$56.88(15)$

$56.21(12)$

29.81 (13)

$107.6(2)$

$123.6(3)$

$128.7(2)$

72.95 (14)

$75.17(14)$

$121.43(16)$

$107.6(2)$

$126.8(2)$

$125.4(2)$

$73.67(14)$

$73.68(13)$

$122.26(17)$

$108.0(2)$

$123.8(2)$

$127.9(2)$

$73.43(13)$

$74.95(13)$

121.25 (16)

$107.3(2)$

$125.2(2)$

$127.2(2)$

73.66 (13)

$75.20(13)$

121.75 (16)

$109.5(2)$

75.84 (14)

$74.99(14)$

$106.7(2)$

$125.4(3)$

$127.7(2)$

72.71 (14)

$74.65(14)$

$121.56(17)$

$108.8(2)$

$123.6(3)$

$127.4(3)$

74.36 (14)

$73.77(14)$

121.82 (17)

107.1(2)

$126.9(2)$

$125.9(3)$

73.24 (13)

$74.79(14)$

120.14 (16)

$107.4(2)$

$123.7(2)$

$128.6(2)$ 


\begin{tabular}{|c|c|}
\hline $\mathrm{C}(10)-\mathrm{C}(9)-\mathrm{Ca}(2)$ & $74.00(13)$ \\
\hline $\mathrm{C}(8)-\mathrm{C}(9)-\mathrm{Ca}(2)$ & $75.37(13)$ \\
\hline $\mathrm{C}(91)-\mathrm{C}(9)-\mathrm{Ca}(2)$ & $121.33(16)$ \\
\hline$C(6)-C(10)-C(9)$ & $110.0(2)$ \\
\hline $\mathrm{C}(6)-\mathrm{C}(10)-\mathrm{Ca}(2)$ & $76.22(14)$ \\
\hline $\mathrm{C}(9)-\mathrm{C}(10)-\mathrm{Ca}(2)$ & $74.61(13)$ \\
\hline$C(12)-C(11)-C(13)$ & $109.5(3)$ \\
\hline$C(12)-C(11)-C(1)$ & $112.9(2)$ \\
\hline$C(13)-C(11)-C(1)$ & $112.0(2)$ \\
\hline$C(2)-C(21)-C(22)$ & $113.6(3)$ \\
\hline$C(2)-C(21)-C(23)$ & $112.0(3)$ \\
\hline$C(22)-C(21)-C(23)$ & $112.4(3)$ \\
\hline$C(32)-C(31)-C(33)$ & $112.1(3)$ \\
\hline$C(32)-C(31)-C(3)$ & $115.3(2)$ \\
\hline$C(33)-C(31)-C(3)$ & $112.8(2)$ \\
\hline$C(4)-C(41)-C(42)$ & $113.5(2)$ \\
\hline$C(4)-C(41)-C(43)$ & $110.3(2)$ \\
\hline$C(42)-C(41)-C(43)$ & $109.8(2)$ \\
\hline$C(52)-C(51)-C(58)$ & $135.8(3)$ \\
\hline$C(52)-C(51)-C a(1)$ & $75.6(2)$ \\
\hline $\mathrm{C}(58)-\mathrm{C}(51)-\mathrm{Ca}(1)$ & 75.11 (19) \\
\hline $\mathrm{C}(52)-\mathrm{C}(51)-\mathrm{Ca}(2)$ & 74.68 (19) \\
\hline $\mathrm{C}(58)-\mathrm{C}(51)-\mathrm{Ca}(2)$ & 75.91 (18) \\
\hline $\mathrm{Ca}(1)-\mathrm{C}(51)-\mathrm{Ca}(2)$ & $95.44(10)$ \\
\hline$C(51)-C(52)-C(53)$ & $135.4(3)$ \\
\hline$C(55)-C(56)-C a(1)$ & $75.5(2)$ \\
\hline$C(57)-C(56)-C a(2)$ & 75.07 (19) \\
\hline$C(55)-C(56)-C a(2)$ & $74.3(2)$ \\
\hline $\mathrm{Ca}(1)-\mathrm{C}(56)-\mathrm{Ca}(2)$ & $95.10(10)$ \\
\hline$C(58)-C(57)-C(56)$ & $135.2(3)$ \\
\hline C (58)-C(57) -Ca (1) & $74.87(18)$ \\
\hline$C(56)-C(57)-C a(1)$ & $74.9(2)$ \\
\hline $\mathrm{C}(58)-\mathrm{C}(57)-\mathrm{Ca}(2)$ & 75.41 (18) \\
\hline $\mathrm{C}(56)-\mathrm{C}(57)-\mathrm{Ca}(2)$ & $75.1(2)$ \\
\hline $\mathrm{Ca}(1)-\mathrm{C}(57)-\mathrm{Ca}$ (2) & $95.06(10)$ \\
\hline$C(51)-C(58)-C(57)$ & $135.3(3)$ \\
\hline$C(51)-C(58)-C a(1)$ & 75.32 (19) \\
\hline $\mathrm{C}(57)-\mathrm{C}(58)-\mathrm{Ca}(1)$ & 75.46 (19) \\
\hline $\mathrm{C}(51)-\mathrm{C}(58)-\mathrm{Ca}(2)$ & $74.67(18)$ \\
\hline$C(92)-C(91)-C(9)$ & $113.3(2)$ \\
\hline
\end{tabular}

$\begin{array}{lc}C(51)-C(52)-C a(2) & 75.90(18) \\ C(53)-C(52)-C a(2) & 74.6(2) \\ C(51)-C(52)-C a(1) & 75.07(19) \\ C(53)-C(52)-C a(1) & 75.4(2) \\ C a(2)-C(52)-C a(1) & 95.64(10) \\ C(52)-C(53)-C(54) & 134.9(4) \\ C(52)-C(53)-C a(2) & 75.5(2) \\ C(54)-C(53)-C a(2) & 74.7(2) \\ C(52)-C(53)-C a(1) & 74.9(2) \\ C(54)-C(53)-C a(1) & 75.3(2) \\ C a(2)-C(53)-C a(1) & 95.75(11) \\ C(55)-C(54)-C(53) & 133.8(4) \\ C(55)-C(54)-C a(2) & 75.4(2) \\ C(53)-C(54)-C a(2) & 74.5(2) \\ C(55)-C(54)-C a(1) & 74.4(2) \\ C(53)-C(54)-C a(1) & 74.4(2) \\ C a(2)-C(54)-C a(1) & 95.44(12) \\ C(56)-C(55)-C(54) & 134.8(4) \\ C(56)-C(55)-C a(2) & 75.8(2) \\ C(54)-C(55)-C a(2) & 74.1(2) \\ C(56)-C(55)-C a(1) & 74.7(2) \\ C(54)-C(55)-C a(1) & 75.4(2) \\ C a(2)-C(55)-C a(1) & 95.32(12) \\ C(57)-C(56)-C(55) & 134.9(3) \\ C(57)-C(56)-C a(1) & 75.12(19) \\ C(57)-C(58)-C a(2) & 75.11(19) \\ C a(1)-C(58)-C a(2) & 95.14(11) \\ C(63)-C(61)-C(6) & 111.5(3) \\ C(63)-C(61)-C(62) & 108.1(3) \\ C(6)-C(61)-C(62) & 112.3(3) \\ C(72)-C(71)-C(73) & 118.0(3) \\ C(72)-C(71)-C(7) & 117.6(3) \\ C(73)-C(71)-C(7) & 114.8(2) \\ C(82)-C(81)-C(83) & 116.7(3) \\ C(82)-C(81)-C(8) & 116.5(3) \\ C(83)-C(81)-C(8) & 114.9(3) \\ C(93)-C(91)-C(92) & 109.4(3) \\ C(93)-C(91)-C(9) & 111.2(2) \\ & \\ C & \\ C & \end{array}$




\section{2. $\left[\left(\mathrm{C}_{5} \mathrm{H}\left(\mathrm{CHMe}_{2}\right)_{4}\right) \mathrm{Ba}\left(\mu, \eta^{8}: \eta^{8}-\mathrm{C}_{8} \mathrm{H}_{8}\right) \mathrm{Ba}\left(\mathrm{C}_{5} \mathrm{H}\left(\mathrm{CHMe}_{2}\right)_{4}\right)\right] \quad\left(2-\mathrm{Ba}^{2} * 2 \mathrm{C}_{6} \mathrm{D}_{6}\right.$}

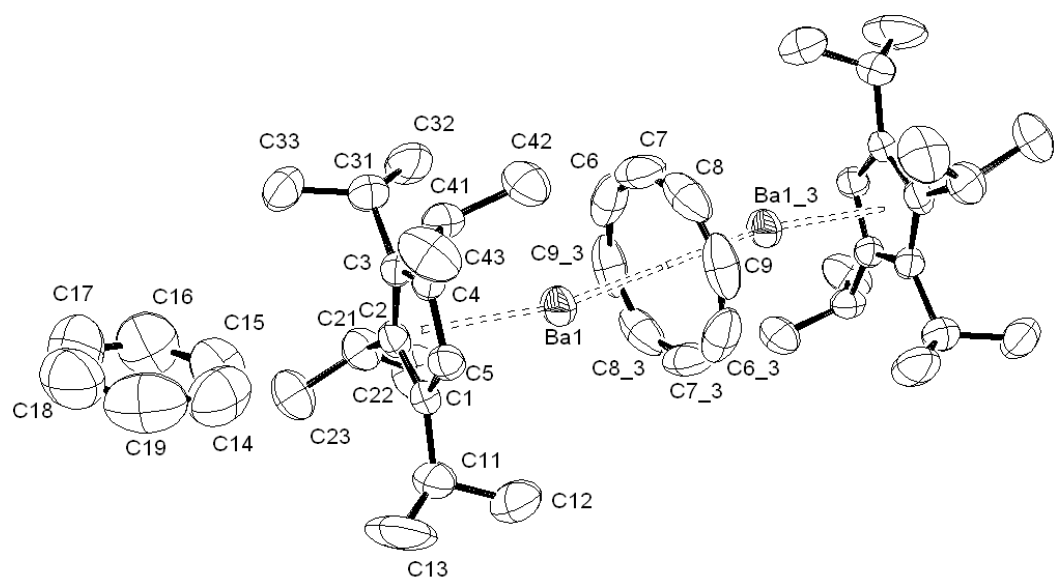

Figure S2. ORTEP diagram of $\left[\left(\mathrm{C}_{5} \mathrm{H}\left(\mathrm{CHMe}_{2}\right)_{4}\right) \mathrm{Ba}\left(\mu, \eta^{8}: \eta^{8}-\mathrm{C}_{8} \mathrm{H}_{8}\right) \mathrm{Ba}\left(\mathrm{C}_{5} \mathrm{H}\left(\mathrm{CHMe}_{2}\right)_{4}\right)\right] \quad(50 \%$ probability ellipsoids). Symmetry related positions are labeled with _3 (-x+1,-y,-z+2)

2.1 Positional Parameters of $\left[\left(\mathrm{C}_{5} \mathrm{H}\left(\mathrm{CHMe}_{2}\right)_{4}\right) \mathrm{Ba}\left(\mu, \eta^{8}: \eta^{8}-\mathrm{C}_{8} \mathrm{H}_{8}\right) \mathrm{Ba}\left(\mathrm{C}_{5} \mathrm{H}\left(\mathrm{CHMe}_{2}\right)_{4}\right)\right]$ 2.1.1 Positional Parameters of Non-Hydrogen atoms of $\left[\left(\mathrm{C}_{5} \mathrm{H}\left(\mathrm{CHMe}_{2}\right)_{4}\right) \mathrm{Ba}\left(\mu, \eta^{8}: \eta^{8}-\mathrm{C}_{8} \mathrm{H}_{8}\right) \mathrm{Ba}\left(\mathrm{C}_{5} \mathrm{H}\left(\mathrm{CHMe}_{2}\right)_{4}\right)\right]$

\begin{tabular}{lcrrr}
\hline & \multicolumn{1}{c}{$\mathrm{Y}$} & $\mathrm{Z}$ & $\mathrm{U}(\mathrm{eq})$ \\
\hline $\mathrm{Ba}(1)$ & & & & \\
$\mathrm{C}(1)$ & $5951(1)$ & $1201(1)$ & $9335(1)$ & $54(1)$ \\
$\mathrm{C}(2)$ & $7456(2)$ & $1762(5)$ & $8629(2)$ & $40(1)$ \\
$\mathrm{C}(3)$ & $7773(2)$ & $2497(5)$ & $9293(2)$ & $42(1)$ \\
$\mathrm{C}(4)$ & $7208(2)$ & $3765(5)$ & $9299(2)$ & $41(1)$ \\
$\mathrm{C}(5)$ & $6556(2)$ & $3834(5)$ & $8642(2)$ & $39(1)$ \\
$\mathrm{C}(6)$ & $6714(3)$ & $2605(5)$ & $8243(2)$ & $42(1)$ \\
$\mathrm{C}(7)$ & $5486(5)$ & $1162(10)$ & $10752(3)$ & $88(2)$ \\
$\mathrm{C}(8)$ & $4720(6)$ & $1789(6)$ & $10313(4)$ & $82(2)$ \\
$\mathrm{C}(9)$ & $4106(4)$ & $1398(8)$ & $9701(4)$ & $82(2)$ \\
$\mathrm{C}(11)$ & $4015(3)$ & $175(10)$ & $9254(3)$ & $82(2)$ \\
$\mathrm{C}(12)$ & $7817(3)$ & $396(6)$ & $8356(3)$ & $61(1)$ \\
$\mathrm{C}(13)$ & $7198(4)$ & $-936(6)$ & $8340(4)$ & $92(2)$ \\
$\mathrm{C}(21)$ & $7988(5)$ & $654(8)$ & $7625(4)$ & $118(3)$ \\
$\mathrm{C}(22)$ & $8597(3)$ & $2070(6)$ & $9880(2)$ & $58(1)$ \\
$\mathrm{C}(23)$ & $8490(4)$ & $598(7)$ & $10220(3)$ & $89(2)$ \\
$\mathrm{C}(31)$ & $9504(3)$ & $2210(7)$ & $9674(3)$ & $77(2)$ \\
$\mathrm{C}(32)$ & $7290(3)$ & $4935(5)$ & $9874(2)$ & $54(1)$ \\
$\mathrm{C}(33)$ & $7120(4)$ & $4396(6)$ & $10557(3)$ & $77(2)$ \\
$\mathrm{C}(41)$ & $8144(4)$ & $5884(6)$ & $9967(3)$ & $47(2)$ \\
$\mathrm{C}(42)$ & $5817(3)$ & $4993(5)$ & $8401(2)$ & $66(1)$ \\
$\mathrm{C}(43)$ & $4909(3)$ & $4581(6)$ & $8585(3)$ & $81(2)$ \\
$\mathrm{C}(14)$ & $5651(4)$ & $5330(7)$ & $7616(3)$ & $92(2)$ \\
$\mathrm{C}(15)$ & $8340(5)$ & $5369(9)$ & $7363(4)$ & $129(2)$ \\
$\mathrm{C}(16)$ & $8692(5)$ & $5224(8)$ & $8048(4)$ & $128(3)$ \\
$\mathrm{C}(17)$ & $9402(5)$ & $6058(10)$ & $8371(4)$ & \\
$\mathrm{C}(18)$ & $9780(5)$ & $7046(11)$ & $8016(6)$ & \\
$\mathrm{C}(19)$ & $9425(8)$ & $7232(10)$ & $7300(6)$ & \\
& $8703(7)$ & $6388(12)$ & $6978(4)$ & \\
\hline
\end{tabular}

2.1.2 Positional Parameters of Hydrogen atoms of $\left[\left(\mathrm{C}_{5} \mathrm{H}\left(\mathrm{CHMe}_{2}\right)_{4}\right) \mathrm{Ba}\left(\mu, \eta^{8}: \eta^{8}-\mathrm{C}_{8} \mathrm{H}_{8}\right) \mathrm{Ba}\left(\mathrm{C}_{5} \mathrm{H}\left(\mathrm{CHMe}_{2}\right)_{4}\right)\right]$ 


\begin{tabular}{|c|c|c|c|c|}
\hline & $\mathrm{x}$ & $\mathrm{Y}$ & z & $\mathrm{U}$ (eq) \\
\hline $\mathrm{H}(5)$ & 6376 & 2382 & 7787 & 51 \\
\hline $\mathrm{H}(6)$ & 5735 & 1749 & 11144 & 105 \\
\hline $\mathrm{H}(7)$ & 4587 & 2714 & 10475 & 99 \\
\hline $\mathrm{H}(8)$ & 3648 & 2103 & 9549 & 98 \\
\hline $\mathrm{H}(9)$ & 3509 & 250 & 8869 & 98 \\
\hline $\mathrm{H}(11)$ & 8412 & 163 & 8678 & 73 \\
\hline $\mathrm{H}(12 \mathrm{~A})$ & 7192 & -1212 & 8815 & 138 \\
\hline $\mathrm{H}(12 \mathrm{~B})$ & 7426 & -1747 & 8111 & 138 \\
\hline $\mathrm{H}(12 \mathrm{C})$ & 6584 & -693 & 8082 & 138 \\
\hline $\mathrm{H}(13 \mathrm{~A})$ & 7426 & 966 & 7305 & 177 \\
\hline $\mathrm{H}(13 \mathrm{~B})$ & 8197 & -250 & 7454 & 177 \\
\hline $\mathrm{H}(13 \mathrm{C})$ & 8449 & 1407 & 7653 & 177 \\
\hline $\mathrm{H}(21)$ & 8621 & 2808 & 10254 & 69 \\
\hline $\mathrm{H}(22 \mathrm{~A})$ & 7983 & 648 & 10443 & 133 \\
\hline $\mathrm{H}(22 \mathrm{~B})$ & 9045 & 372 & 10570 & 133 \\
\hline $\mathrm{H}(22 \mathrm{C})$ & 8375 & -162 & 9864 & 133 \\
\hline$H(23 A)$ & 10000 & 2017 & 10080 & 115 \\
\hline $\mathrm{H}(23 \mathrm{~B})$ & 9564 & 3195 & 9504 & 115 \\
\hline $\mathrm{H}(23 \mathrm{C})$ & 9525 & 1508 & 9307 & 115 \\
\hline $\mathrm{H}(31)$ & 6779 & 5618 & 9689 & 64 \\
\hline $\mathrm{H}(32 \mathrm{~A})$ & 7593 & 3705 & 10771 & 116 \\
\hline $\mathrm{H}(32 \mathrm{~B})$ & 6531 & 3916 & 10470 & 116 \\
\hline $\mathrm{H}(32 \mathrm{C})$ & 7128 & 5220 & 10871 & 116 \\
\hline $\mathrm{H}(33 \mathrm{~A})$ & 8101 & 6708 & 10269 & 109 \\
\hline $\mathrm{H}(33 \mathrm{~B})$ & 8197 & 6243 & 9513 & 109 \\
\hline $\mathrm{H}(33 \mathrm{C})$ & 8678 & 5303 & 10177 & 109 \\
\hline $\mathrm{H}(41)$ & 6036 & 5908 & 8657 & 56 \\
\hline $\mathrm{H}(42 \mathrm{~A})$ & 4656 & 3714 & 8323 & 99 \\
\hline $\mathrm{H}(42 \mathrm{~B})$ & 4482 & 5388 & 8463 & 99 \\
\hline $\mathrm{H}(42 \mathrm{C})$ & 5019 & 4384 & 9083 & 99 \\
\hline $\mathrm{H}(43 \mathrm{~A})$ & 6217 & 5646 & 7511 & 121 \\
\hline $\mathrm{H}(43 \mathrm{~B})$ & 5200 & 6102 & 7492 & 121 \\
\hline $\mathrm{H}(43 \mathrm{C})$ & 5432 & 4455 & 7348 & 121 \\
\hline $\mathrm{H}(14)$ & 7845 & 4782 & 7138 & 126 \\
\hline $\mathrm{H}(15)$ & 8444 & 4536 & 8307 & 110 \\
\hline $\mathrm{H}(16)$ & 9641 & 5951 & 8856 & 130 \\
\hline $\mathrm{H}(17)$ & 10283 & 7607 & 8251 & 155 \\
\hline $\mathrm{H}(18)$ & 9676 & 7923 & 7045 & 154 \\
\hline $\mathrm{H}(19)$ & 8450 & 6493 & 6494 & 144 \\
\hline
\end{tabular}

2.2 Thermal Parameters of $\left[\left(\mathrm{C}_{5} \mathrm{H}\left(\mathrm{CHMe}_{2}\right)_{4}\right) \mathrm{Ba}\left(\mu, \eta^{8}: \eta^{8}-\mathrm{C}_{8} \mathrm{H}_{8}\right) \mathrm{Ba}\left(\mathrm{C}_{5} \mathrm{H}\left(\mathrm{CHMe}_{2}\right)_{4}\right)\right]$

\begin{tabular}{|c|c|c|c|c|c|c|}
\hline & $\mathrm{U}_{11}$ & $\mathrm{U}_{22}$ & $\mathrm{U}_{33}$ & $\mathrm{U}_{23}$ & $\mathrm{U}_{13}$ & $\mathrm{U}_{12}$ \\
\hline $\mathrm{Ba}(1)$ & $56(1)$ & 49 (1) & 65 (1) & $5(1)$ & $30(1)$ & $-6(1)$ \\
\hline$C(1)$ & $31(2)$ & $41(3)$ & $50(2)$ & $-4(2)$ & $14(2)$ & $-4(2)$ \\
\hline$C(2)$ & $30(2)$ & $53(3)$ & $43(2)$ & $-2(2)$ & $9(2)$ & $-3(2)$ \\
\hline$C(3)$ & $40(2)$ & $43(3)$ & $41(2)$ & $-6(2)$ & $13(2)$ & $-7(2)$ \\
\hline$C(4)$ & $38(2)$ & $38(2)$ & $40(2)$ & $-1(2)$ & $11(2)$ & $-4(2)$ \\
\hline$C(5)$ & $42(2)$ & $50(3)$ & $35(2)$ & $-6(2)$ & $12(2)$ & $-2(2)$ \\
\hline$C(6)$ & $116(5)$ & $107(6)$ & $50(3)$ & $-29(4)$ & $38(3)$ & $-67(5)$ \\
\hline$C(7)$ & $131(6)$ & $37(4)$ & $105(5)$ & $-8(3)$ & $80(5)$ & $-5(4)$ \\
\hline$C(8)$ & $65(3)$ & $72(5)$ & $125(6)$ & $46(4)$ & $54(4)$ & $28(3)$ \\
\hline$C(9)$ & $42(3)$ & $127(7)$ & $69(4)$ & $37(4)$ & $-1(2)$ & $-18(3)$ \\
\hline$C(11)$ & $51(3)$ & $60(4)$ & $73(3)$ & $-13(3)$ & $18(2)$ & $7(2)$ \\
\hline C (12) & $92(4)$ & $60(5)$ & $125(5)$ & $-29(4)$ & $28(4)$ & $3(3)$ \\
\hline$C(13)$ & $165(7)$ & $112(6)$ & $107(5)$ & $-28(4)$ & $91(5)$ & $26(5)$ \\
\hline C (21) & $46(3)$ & $66(4)$ & $57(3)$ & $6(2)$ & $5(2)$ & $7(2)$ \\
\hline C (22) & $76(4)$ & $85(5)$ & $97(4)$ & $37(4)$ & $7(3)$ & $18(3)$ \\
\hline C (23) & $40(3)$ & $98(5)$ & $89(4)$ & $6(3)$ & $9(3)$ & $8(3)$ \\
\hline C (31) & $59(3)$ & $53(3)$ & $50(3)$ & $-9(2)$ & $16(2)$ & $-2(2)$ \\
\hline C (32) & $108(4)$ & $74(4)$ & $60(3)$ & $-23(3)$ & $39(3)$ & $-18(3)$ \\
\hline C ( 33 ) & $84(4)$ & $61(5)$ & 75 (3) & $-21(3)$ & $26(3)$ & $-24(3)$ \\
\hline
\end{tabular}




\begin{tabular}{rrrrrrr}
$C(41)$ & $49(2)$ & $43(3)$ & $48(2)$ & $-4(2)$ & $11(2)$ & $6(2)$ \\
$\mathrm{C}(42)$ & $52(3)$ & $70(4)$ & $76(3)$ & $5(3)$ & $17(2)$ & $15(2)$ \\
$\mathrm{C}(43)$ & $76(4)$ & $97(5)$ & $73(3)$ & $31(3)$ & $24(3)$ & $36(3)$ \\
$\mathrm{C}(14)$ & $88(5)$ & $101(6)$ & $110(6)$ & $-21(5)$ & $-6(4)$ & $1(4)$ \\
$\mathrm{C}(15)$ & $85(4)$ & $104(6)$ & $89(5)$ & $8(4)$ & $27(4)$ & $1(4)$ \\
$\mathrm{C}(16)$ & $106(5)$ & $135(7)$ & $80(4)$ & $-2(5)$ & $14(4)$ & $-6(5)$ \\
$\mathrm{C}(17)$ & $97(6)$ & $142(9)$ & $149(8)$ & $-33(7)$ & $29(6)$ & $-38(5)$ \\
$\mathrm{C}(18)$ & $166(9)$ & $97(7)$ & $154(9)$ & $18(6)$ & $99(7)$ & $5(6)$ \\
$\mathrm{C}(19)$ & $170(8)$ & $117(8)$ & $74(4)$ & $12(5)$ & $30(5)$ & $37(7)$ \\
\hline
\end{tabular}

2.3 Bond distances $(\AA)$ for $\left[\left(\mathrm{C}_{5} \mathrm{H}\left(\mathrm{CHMe}_{2}\right)_{4}\right) \mathrm{Ba}\left(\mu, \eta^{8}: \eta^{8}-\mathrm{C}_{8} \mathrm{H}_{8}\right) \mathrm{Ba}\left(\mathrm{C}_{5} \mathrm{H}\left(\mathrm{CHMe}_{2}\right)_{4}\right)\right]$

\begin{tabular}{llll}
\hline $\mathrm{Ba}(1)-\mathrm{C}(5)$ & $2.927(4)$ & $\mathrm{C}(6)-\mathrm{C}(9) \# 1$ & $1.420(10)$ \\
$\mathrm{Ba}(1)-\mathrm{C}(1)$ & $2.945(4)$ & $\mathrm{C}(6)-\mathrm{Ba}(1) \# 1$ & $3.003(5)$ \\
$\mathrm{Ba}(1)-\mathrm{C}(4)$ & $2.977(4)$ & $\mathrm{C}(7)-\mathrm{C}(8)$ & $1.364(9)$ \\
$\mathrm{Ba}(1)-\mathrm{C}(2)$ & $2.982(4)$ & $\mathrm{C}(7)-\mathrm{Ba}(1) \# 1$ & $3.015(5)$ \\
$\mathrm{Ba}(1)-\mathrm{C}(3)$ & $2.993(4)$ & $\mathrm{C}(8)-\mathrm{C}(9)$ & $1.391(10)$ \\
$\mathrm{Ba}(1)-\mathrm{C}(7)$ & $2.994(5)$ & $\mathrm{C}(8)-\mathrm{Ba}(1) \# 1$ & $3.016(6)$ \\
$\mathrm{Ba}(1)-\mathrm{C}(9) \# 1$ & $3.000(5)$ & $\mathrm{C}(9)-\mathrm{C}(6) \# 1$ & $1.420(10)$ \\
$\mathrm{Ba}(1)-\mathrm{C}(6)$ & $2.998(4)$ & $\mathrm{C}(9)-\mathrm{Ba}(1) \# 1$ & $3.000(5)$ \\
$\mathrm{Ba}(1)-\mathrm{C}(6) \# 1$ & $3.003(5)$ & $\mathrm{C}(11)-\mathrm{C}(12)$ & $1.513(7)$ \\
$\mathrm{Ba}(1)-\mathrm{C}(9)$ & $3.007(5)$ & $\mathrm{C}(11)-\mathrm{C}(13)$ & $1.521(7)$ \\
$\mathrm{Ba}(1)-\mathrm{C}(7) \# 1$ & $3.015(6)$ & $\mathrm{C}(21)-\mathrm{C}(22)$ & $1.510(7)$ \\
$\mathrm{Ba}(1)-\mathrm{C}(8)$ & $3.014(4)$ & $\mathrm{C}(21)-\mathrm{C}(23)$ & $1.508(6)$ \\
$\mathrm{C}(1)-\mathrm{C}(5)$ & $1.405(6)$ & $\mathrm{C}(31)-\mathrm{C}(32)$ & $1.492(6)$ \\
$\mathrm{C}(1)-\mathrm{C}(2)$ & $1.427(6)$ & $\mathrm{C}(31)-\mathrm{C}(33)$ & $1.513(6)$ \\
$\mathrm{C}(1)-\mathrm{C}(11)$ & $1.494(6)$ & $\mathrm{C}(41)-\mathrm{C}(43)$ & $1.517(6)$ \\
$\mathrm{C}(2)-\mathrm{C}(3)$ & $1.425(6)$ & $\mathrm{C}(41)-\mathrm{C}(42)$ & $1.529(6)$ \\
$\mathrm{C}(2)-\mathrm{C}(21)$ & $1.515(6)$ & $\mathrm{C}(14)-\mathrm{C}(15)$ & $1.315(9)$ \\
$\mathrm{C}(3)-\mathrm{C}(4)$ & $1.408(5)$ & $\mathrm{C}(14)-\mathrm{C}(19)$ & $1.376(11)$ \\
$\mathrm{C}(3)-\mathrm{C}(31)$ & $1.521(6)$ & $\mathrm{C}(15)-\mathrm{C}(16)$ & $1.330(9)$ \\
$\mathrm{C}(4)-\mathrm{C}(5)$ & $1.406(6)$ & $\mathrm{C}(16)-\mathrm{C}(17)$ & $1.334(11)$ \\
$\mathrm{C}(4)-\mathrm{C}(41)$ & $1.513(6)$ & $\mathrm{C}(17)-\mathrm{C}(18)$ & $1.374(11)$ \\
$\mathrm{C}(6)-\mathrm{C}(7)$ & $1.376(10)$ & $\mathrm{C}(18)-\mathrm{C}(19)$ & $1.347(11)$ \\
& & & \\
\hline
\end{tabular}

Symmetry equivalent positions \#1 -x+1,-y,-z+2 
2.4 Bond angles $\left(^{\circ}\right)$ for $\left[\left(\mathrm{C}_{5} \mathrm{H}\left(\mathrm{CHMe}_{2}\right)_{4}\right) \mathrm{Ba}\left(\mu, \eta^{8}: \eta^{8}-\mathrm{C}_{8} \mathrm{H}_{8}\right) \mathrm{Ba}\left(\mathrm{C}_{5} \mathrm{H}\left(\mathrm{CHMe}_{2}\right)_{4}\right)\right]$

\begin{tabular}{|c|c|c|c|}
\hline $\mathrm{C}(5)-\mathrm{Ba}(1)-\mathrm{C}(1)$ & $27.68(11)$ & $\mathrm{C}(7) \# 1-\mathrm{Ba}(1)-\mathrm{C}(8)$ & $68.06(16)$ \\
\hline $\mathrm{C}(5)-\mathrm{Ba}(1)-\mathrm{C}(4)$ & $27.53(11)$ & $C(5)-C(1)-C(2)$ & $106.3(4)$ \\
\hline $\mathrm{C}(1)-\mathrm{Ba}(1)-\mathrm{C}(4)$ & $45.86(11)$ & $C(5)-C(1)-C(11)$ & $124.3(4)$ \\
\hline $\mathrm{C}(5)-\mathrm{Ba}(1)-\mathrm{C}(2)$ & $45.07(11)$ & $C(2)-C(1)-C(11)$ & $129.4(4)$ \\
\hline $\mathrm{C}(1)-\mathrm{Ba}(1)-\mathrm{C}(2)$ & $27.85(11)$ & $\mathrm{C}(5)-\mathrm{C}(1)-\mathrm{Ba}(1)$ & $75.5(2)$ \\
\hline $\mathrm{C}(4)-\mathrm{Ba}(1)-\mathrm{C}(2)$ & $45.28(11)$ & $\mathrm{C}(2)-\mathrm{C}(1)-\mathrm{Ba}(1)$ & $77.5(2)$ \\
\hline $\mathrm{C}(5)-\mathrm{Ba}(1)-\mathrm{C}(3)$ & $44.95(11)$ & $\mathrm{C}(11)-\mathrm{C}(1)-\mathrm{Ba}(1)$ & $113.8(3)$ \\
\hline $\mathrm{C}(1)-\mathrm{Ba}(1)-\mathrm{C}(3)$ & $45.76(11)$ & $C(1)-C(2)-C(3)$ & $108.2(3)$ \\
\hline $\mathrm{C}(4)-\mathrm{Ba}(1)-\mathrm{C}(3)$ & $27.29(10)$ & $C(1)-C(2)-C(21)$ & $126.6(4)$ \\
\hline $\mathrm{C}(2)-\mathrm{Ba}(1)-\mathrm{C}(3)$ & $27.59(11)$ & $C(3)-C(2)-C(21)$ & $125.2(4)$ \\
\hline $\mathrm{C}(5)-\mathrm{Ba}(1)-\mathrm{C}(7)$ & $142.09(15)$ & $\mathrm{C}(1)-\mathrm{C}(2)-\mathrm{Ba}(1)$ & $74.6(2)$ \\
\hline $\mathrm{C}(1)-\mathrm{Ba}(1)-\mathrm{C}(7)$ & $156.85(15)$ & $\mathrm{C}(3)-\mathrm{C}(2)-\mathrm{Ba}(1)$ & $76.6(2)$ \\
\hline $\mathrm{C}(4)-\mathrm{Ba}(1)-\mathrm{C}(7)$ & $116.41(13)$ & $\mathrm{C}(21)-\mathrm{C}(2)-\mathrm{Ba}(1)$ & $117.6(3)$ \\
\hline $\mathrm{C}(2)-\mathrm{Ba}(1)-\mathrm{C}(7)$ & $130.68(17)$ & $C(4)-C(3)-C(2)$ & $108.1(4)$ \\
\hline $\mathrm{C}(3)-\mathrm{Ba}(1)-\mathrm{C}(7)$ & $111.24(14)$ & $C(4)-C(3)-C(31)$ & $123.5(4)$ \\
\hline $\mathrm{C}(5)-\mathrm{Ba}(1)-\mathrm{C}(9) \# 1$ & $156.60(12)$ & $C(2)-C(3)-C(31)$ & $128.3(4)$ \\
\hline $\mathrm{C}(1)-\mathrm{Ba}(1)-\mathrm{C}(9) \# 1$ & $130.49(13)$ & $\mathrm{C}(4)-\mathrm{C}(3)-\mathrm{Ba}(1)$ & $75.7(2)$ \\
\hline $\mathrm{C}(4)-\mathrm{Ba}(1)-\mathrm{C}(9) \# 1$ & $143.45(17)$ & $\mathrm{C}(2)-\mathrm{C}(3)-\mathrm{Ba}(1)$ & $75.8(2)$ \\
\hline $\mathrm{C}(2)-\mathrm{Ba}(1)-\mathrm{C}(9) \# 1$ & $111.82(12)$ & $\mathrm{C}(31)-\mathrm{C}(3)-\mathrm{Ba}(1)$ & $117.4(2)$ \\
\hline $\mathrm{C}(3)-\mathrm{Ba}(1)-\mathrm{C}(9) \# 1$ & $117.94(15)$ & $C(5)-C(4)-C(3)$ & $107.1(4)$ \\
\hline $\mathrm{C}(7)-\mathrm{Ba}(1)-\mathrm{C}(9) \# 1$ & $51.0(2)$ & $C(5)-C(4)-C(41)$ & $125.6(3)$ \\
\hline $\mathrm{C}(5)-\mathrm{Ba}(1)-\mathrm{C}(6)$ & $152.55(15)$ & $C(3)-C(4)-C(41)$ & $127.3(4)$ \\
\hline $\mathrm{C}(1)-\mathrm{Ba}(1)-\mathrm{C}(6)$ & $143.21(14)$ & $\mathrm{C}(5)-\mathrm{C}(4)-\mathrm{Ba}(1)$ & $74.3(2)$ \\
\hline $\mathrm{C}(4)-\mathrm{Ba}(1)-\mathrm{C}(6)$ & 125.95 (17) & $\mathrm{C}(3)-\mathrm{C}(4)-\mathrm{Ba}(1)$ & $77.0(2)$ \\
\hline $\mathrm{C}(2)-\mathrm{Ba}(1)-\mathrm{C}(6)$ & $116.34(13)$ & $\mathrm{C}(41)-\mathrm{C}(4)-\mathrm{Ba}(1)$ & $114.4(2)$ \\
\hline $\mathrm{C}(3)-\mathrm{Ba}(1)-\mathrm{C}(6)$ & $108.40(13)$ & $C(1)-C(5)-C(4)$ & $110.3(3)$ \\
\hline $\mathrm{C}(7)-\mathrm{Ba}(1)-\mathrm{C}(6)$ & 26.55 (19) & $\mathrm{C}(1)-\mathrm{C}(5)-\mathrm{Ba}(1)$ & $76.9(2)$ \\
\hline $\mathrm{C}(9) \# 1-\mathrm{Ba}(1)-\mathrm{C}(6)$ & $27.39(19)$ & $\mathrm{C}(4)-\mathrm{C}(5)-\mathrm{Ba}(1)$ & $78.2(2)$ \\
\hline $\mathrm{C}(5)-\mathrm{Ba}(1)-\mathrm{C}(6) \# 1$ & $131.63(12)$ & $C(7)-C(6)-C(9) \# 1$ & $134.9(6)$ \\
\hline $\mathrm{C}(1)-\mathrm{Ba}(1)-\mathrm{C}(6) \# 1$ & $135.38(15)$ & $C(7)-C(6)-B a(1)$ & $76.6(3)$ \\
\hline $\mathrm{C}(4)-\mathrm{Ba}(1)-\mathrm{C}(6) \# 1$ & $146.32(16)$ & $\mathrm{C}(9) \# 1-\mathrm{C}(6)-\mathrm{Ba}(1)$ & $76.4(3)$ \\
\hline $\mathrm{C}(2)-\mathrm{Ba}(1)-\mathrm{C}(6) \# 1$ & $157.3(2)$ & $\mathrm{C}(7)-\mathrm{C}(6)-\mathrm{Ba}(1) \# 1$ & $77.3(3)$ \\
\hline $\mathrm{C}(3)-\mathrm{Ba}(1)-\mathrm{C}(6) \# 1$ & $173.0(2)$ & $\mathrm{C}(9) \# 1-\mathrm{C}(6)-\mathrm{Ba}(1) \# 1$ & $76.5(3)$ \\
\hline $\mathrm{C}(7)-\mathrm{Ba}(1)-\mathrm{C}(6) \# 1$ & $67.76(16)$ & $\mathrm{Ba}(1)-\mathrm{C}(6)-\mathrm{Ba}(1) \# 1$ & $106.07(16)$ \\
\hline $\mathrm{C}(9) \# 1-\mathrm{Ba}(1)-\mathrm{C}(6) \# 1$ & $67.22(15)$ & $C(8)-C(7)-C(6)$ & $136.1(6)$ \\
\hline $\mathrm{C}(6)-\mathrm{Ba}(1)-\mathrm{C}(6) \# 1$ & $73.93(16)$ & $\mathrm{C}(8)-\mathrm{C}(7)-\mathrm{Ba}(1)$ & $77.7(3)$ \\
\hline $\mathrm{C}(5)-\mathrm{Ba}(1)-\mathrm{C}(9)$ & $129.23(13)$ & $\mathrm{C}(6)-\mathrm{C}(7)-\mathrm{Ba}(1)$ & $76.9(3)$ \\
\hline $\mathrm{C}(1)-\mathrm{Ba}(1)-\mathrm{C}(9)$ & $149.48(15)$ & $\mathrm{C}(8)-\mathrm{C}(7)-\mathrm{Ba}(1) \# 1$ & $77.0(3)$ \\
\hline $\mathrm{C}(4)-\mathrm{Ba}(1)-\mathrm{C}(9)$ & $127.95(14)$ & $\mathrm{C}(6)-\mathrm{C}(7)-\mathrm{Ba}(1) \# 1$ & $76.3(4)$ \\
\hline $\mathrm{C}(2)-\mathrm{Ba}(1)-\mathrm{C}(9)$ & $173.21(15)$ & $\mathrm{Ba}(1)-\mathrm{C}(7)-\mathrm{Ba}(1) \# 1$ & 105.88 (17) \\
\hline $\mathrm{C}(3)-\mathrm{Ba}(1)-\mathrm{C}(9)$ & $146.99(19)$ & $C(7)-C(8)-C(9)$ & $134.1(5)$ \\
\hline $\mathrm{C}(7)-\mathrm{Ba}(1)-\mathrm{C}(9)$ & $50.0(2)$ & $\mathrm{C}(7)-\mathrm{C}(8)-\mathrm{Ba}(1)$ & $76.0(3)$ \\
\hline $\mathrm{C}(9) \# 1-\mathrm{Ba}(1)-\mathrm{C}(9)$ & $74.08(16)$ & $\mathrm{C}(9)-\mathrm{C}(8)-\mathrm{Ba}(1)$ & $76.4(3)$ \\
\hline $\mathrm{C}(6)-\mathrm{Ba}(1)-\mathrm{C}(9)$ & $67.19(15)$ & $\mathrm{C}(7)-\mathrm{C}(8)-\mathrm{Ba}(1) \# 1$ & $76.9(3)$ \\
\hline $\mathrm{C}(6) \# 1-\mathrm{Ba}(1)-\mathrm{C}(9)$ & $27.33(19)$ & $\mathrm{C}(9)-\mathrm{C}(8)-\mathrm{Ba}(1) \# 1$ & $76.0(3)$ \\
\hline $\mathrm{C}(5)-\mathrm{Ba}(1)-\mathrm{C}(7) \# 1$ & $139.52(14)$ & $\mathrm{Ba}(1)-\mathrm{C}(8)-\mathrm{Ba}(1) \# 1$ & $105.33(16)$ \\
\hline $\mathrm{C}(1)-\mathrm{Ba}(1)-\mathrm{C}(7) \# 1$ & $126.33(13)$ & $C(8)-C(9)-C(6) \# 1$ & $134.9(5)$ \\
\hline $\mathrm{C}(4)-\mathrm{Ba}(1)-\mathrm{C}(7) \# 1$ & $166.18(15)$ & $\mathrm{C}(8)-\mathrm{C}(9)-\mathrm{Ba}(1) \# 1$ & $77.3(3)$ \\
\hline $\mathrm{C}(2)-\mathrm{Ba}(1)-\mathrm{C}(7) \# 1$ & $135.48(18)$ & $\mathrm{C}(6) \# 1-\mathrm{C}(9)-\mathrm{Ba}(1) \# 1$ & $76.2(3)$ \\
\hline $\mathrm{C}(3)-\mathrm{Ba}(1)-\mathrm{C}(7) \# 1$ & $160.5(2)$ & $\mathrm{C}(8)-\mathrm{C}(9)-\mathrm{Ba}(1)$ & $76.9(3)$ \\
\hline $\mathrm{C}(7)-\mathrm{Ba}(1)-\mathrm{C}(7) \# 1$ & $74.12(17)$ & $\mathrm{C}(6) \# 1-\mathrm{C}(9)-\mathrm{Ba}(1)$ & $76.2(3)$ \\
\hline $\mathrm{C}(9) \# 1-\mathrm{Ba}(1)-\mathrm{C}(7) \# 1$ & $49.9(2)$ & $\mathrm{Ba}(1) \# 1-\mathrm{C}(9)-\mathrm{Ba}(1)$ & $105.92(16)$ \\
\hline $\mathrm{C}(6)-\mathrm{Ba}(1)-\mathrm{C}(7) \# 1$ & $67.55(16)$ & $C(1)-C(11)-C(12)$ & $113.1(4)$ \\
\hline $\mathrm{C}(6) \# 1-\mathrm{Ba}(1)-\mathrm{C}(7) \# 1$ & $26.43(19)$ & $C(1)-C(11)-C(13)$ & $111.2(5)$ \\
\hline $\mathrm{C}(9)-\mathrm{Ba}(1)-\mathrm{C}(7) \# 1$ & $50.8(2)$ & $C(12)-C(11)-C(13)$ & $109.9(5)$ \\
\hline $\mathrm{C}(5)-\mathrm{Ba}(1)-\mathrm{C}(8)$ & $133.06(14)$ & $C(22)-C(21)-C(2)$ & $113.7(4)$ \\
\hline $\mathrm{C}(1)-\mathrm{Ba}(1)-\mathrm{C}(8)$ & $160.69(13)$ & $C(22)-C(21)-C(23)$ & $112.7(4)$ \\
\hline $\mathrm{C}(4)-\mathrm{Ba}(1)-\mathrm{C}(8)$ & $116.98(14)$ & $C(2)-C(21)-C(23)$ & $113.6(4)$ \\
\hline $\mathrm{C}(2)-\mathrm{Ba}(1)-\mathrm{C}(8)$ & 151.18 (19) & $C(32)-C(31)-C(33)$ & $113.4(4)$ \\
\hline $\mathrm{C}(3)-\mathrm{Ba}(1)-\mathrm{C}(8)$ & $125.08(17)$ & $C(32)-C(31)-C(3)$ & $115.2(4)$ \\
\hline $\mathrm{C}(7)-\mathrm{Ba}(1)-\mathrm{C}(8)$ & $26.24(18)$ & $C(33)-C(31)-C(3)$ & $113.5(4)$ \\
\hline $\mathrm{C}(9) \# 1-\mathrm{Ba}(1)-\mathrm{C}(8)$ & $67.96(15)$ & $C(4)-C(41)-C(43)$ & $112.4(3)$ \\
\hline $\mathrm{C}(6)-\mathrm{Ba}(1)-\mathrm{C}(8)$ & 50.00 (19) & $C(4)-C(41)-C(42)$ & $112.6(4)$ \\
\hline $\mathrm{C}(6) \# 1-\mathrm{Ba}(1)-\mathrm{C}(8)$ & $51.1(2)$ & $C(43)-C(41)-C(42)$ & $110.1(4)$ \\
\hline $\mathrm{C}(9)-\mathrm{Ba}(1)-\mathrm{C}(8)$ & $26.72(19)$ & $C(15)-C(14)-C(19)$ & $120.2(7)$ \\
\hline
\end{tabular}


$\mathrm{C}(14)-\mathrm{C}(15)-\mathrm{C}(16)$

$120.1(7)$

$121.6(7)$

$\mathrm{C}(16)-\mathrm{C}(17)-\mathrm{C}(18)$

$119.7(8)$

$\mathrm{C}(15)-\mathrm{C}(16)-\mathrm{C}(17)$

$120.1(7)$

$\mathrm{C}(19)-\mathrm{C}(18)-\mathrm{C}(17)$

$118.3(8)$

Symmetry equivalent positions \#1 - $\mathrm{x}+1,-\mathrm{y},-\mathrm{z}+2$ 


\section{Selected data collection and structure solution parameters}

\begin{tabular}{|c|c|c|}
\hline Compound & 2-Ca & $(2-B a) * 2 C_{6} D_{6}$ \\
\hline diffractometer & Stoe IPDS & Stoe IPDS \\
\hline space group & $\mathrm{P} 2{ }_{1} / \mathrm{n}$ & $\mathrm{P} 2{ }_{1} / \mathrm{c}$ \\
\hline $\mathrm{a}(\AA)$ & $9.6021(8)$ & $14.9481(14)$ \\
\hline $\mathrm{b}(\AA)$ & $26.5918(15)$ & $9.0286(6)$ \\
\hline$c(\AA)$ & $16.0383(11)$ & $19.430(2)$ \\
\hline$\beta\left(^{\circ}\right)$ & 101.673(9) & $103.846(12)$ \\
\hline$V\left(\AA^{3}\right)$ & $4010.5(5)$ & $2546.0(4)$ \\
\hline $\mathrm{Z}$ & 4 & 4 \\
\hline$d_{\text {calc }}\left(\mathrm{g} / \mathrm{cm}^{3}\right)$ & 1.078 & 1.307 \\
\hline$\mu(\mathrm{Mo}-\mathrm{K} \alpha)_{\mathrm{calc}}$ & 0.310 & 1.569 \\
\hline Size $(\mathrm{mm})$ & $0.56 \times 0.36 \times 0.32$ & $0.48 \times 0.40 \times 0.16$ \\
\hline temperature $(\mathrm{K})$ & 293(2) & 293(2) \\
\hline scan type, $\theta_{\max }$ & $\phi, 25.68$ & $\phi, 25.68$ \\
\hline Reflections integrated & 44377 & 34732 \\
\hline unique reflections, $R_{\text {int }}$ & $7614,0.0904$ & $4729,0.1286$ \\
\hline good reflections & $4018, F_{\mathrm{o}}^{2}>2 \sigma\left(F_{\mathrm{o}}^{2}\right)$ & $2821, F_{\mathrm{o}}^{2}>2 \sigma\left(F_{\mathrm{o}}^{2}\right)$ \\
\hline Variables & 413 & 261 \\
\hline transmission range & $0.91902-088259$ & $0.77349-0.49486$ \\
\hline$R_{1}{ }^{\mathrm{a}}$ & 0.0454 & 0.0345 \\
\hline$w R_{2}{ }^{\mathrm{b}}$ & 0.1028 & 0.0708 \\
\hline$R_{\text {all }}$ & 0.0935 & 0.0694 \\
\hline GOF (on $\mathrm{F}^{2}$ ) & 0.838 & 0.839 \\
\hline $\begin{array}{l}\max / \min \text { peaks in final } \\
\text { difference map }\end{array}$ & $0.382 /-0.263 \mathrm{e}^{-} / \AA^{3}$ & $0.688 /-0.662 \mathrm{e}^{-} / \AA^{3}$ \\
\hline
\end{tabular}

\footnotetext{
${ }^{a} R_{1}=\Sigma|| \mathrm{Fo}|-| \mathrm{Fc} \| / \Sigma|\mathrm{Fo}| \cdot{ }^{b} w R_{2}=\left[\left(\Sigma \mathrm{w}\left(|\mathrm{Fo}|^{2}-|\mathrm{Fc}|^{2}\right)^{2} / \Sigma \mathrm{w}|\mathrm{Fo}|^{4}\right)\right]^{1 / 2}$
}

Structure solution and refinement: SHELXS-97/SHELXL-97 program 


\section{SDD Basis Sets for Ba and Sr (Gaussian Input)}

\subsection{Barium}

\begin{tabular}{|c|c|c|}
\hline $\mathrm{BA}$ & 0 & \\
\hline $\mathrm{S}$ & 31.00 & \\
\hline & 2.39619000 & -5.92889500 \\
\hline & 2.24330500 & 6.64693400 \\
\hline & 0.717402000 & -0.551437000 \\
\hline $\mathrm{S}$ & 11.00 & \\
\hline & 0.278446000 & 1.00000000 \\
\hline S & 11.00 & \\
\hline & $0.431880000 \mathrm{E}-01$ & 1.00000000 \\
\hline $\mathrm{S}$ & 11.00 & \\
\hline & $0.197980000 \mathrm{E}-01$ & 1.00000000 \\
\hline $\mathrm{P}$ & 31.00 & \\
\hline & 2.92674200 & 0.763359000 \\
\hline & 2.52071800 & -1.02201400 \\
\hline & 0.524095000 & 0.649836000 \\
\hline $\mathrm{P}$ & 11.00 & \\
\hline & 0.203428000 & 1.00000000 \\
\hline $\mathrm{P}$ & 11.00 & \\
\hline & $0.479960000 \mathrm{E}-01$ & 1.00000000 \\
\hline $\mathrm{P}$ & 11.00 & \\
\hline & $0.200950000 \mathrm{E}-01$ & 1.00000000 \\
\hline D & 31.00 & \\
\hline & 0.966315000 & -0.908938000 \\
\hline & 0.893828000 & 0.947240000 \\
\hline & 0.273195000 & 0.322057000 \\
\hline D & 21.00 & \\
\hline & 0.103891000 & 0.473260000 \\
\hline & $0.355780000 \mathrm{E}-01$ & 0.365977000 \\
\hline F & 11.00 & \\
\hline & 0.697000000 & 1.00000000 \\
\hline 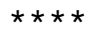 & & \\
\hline a 0 & & \\
\hline DD & & \\
\hline
\end{tabular}

\subsection{Strontium}

\begin{tabular}{|c|c|c|}
\hline SR & 0 & \\
\hline S & 31.00 & \\
\hline & 5.87915700 & 0.196709000 \\
\hline & 3.09248200 & -0.625898000 \\
\hline & 0.644667000 & 0.735723000 \\
\hline S & 11.00 & \\
\hline & 0.298876000 & 1.00000000 \\
\hline$S$ & 11.00 & \\
\hline & $0.572760000 \mathrm{E}-01$ & 1.00000000 \\
\hline$S$ & 11.00 & \\
\hline & $0.238700000 \mathrm{E}-01$ & 1.00000000 \\
\hline $\mathrm{P}$ & 31.00 & \\
\hline
\end{tabular}




\begin{tabular}{|c|c|c|}
\hline & 2.43247200 & -0.374899000 \\
\hline & 1.66423400 & 0.387615000 \\
\hline & 0.569989000 & 0.655838000 \\
\hline $\mathrm{P}$ & 11.00 & \\
\hline & 0.220718000 & 1.00000000 \\
\hline P & 11.00 & \\
\hline & $0.676290000 \mathrm{E}-01$ & 1.00000000 \\
\hline P & 11.00 & \\
\hline & $0.267270000 \mathrm{E}-01$ & 1.00000000 \\
\hline D & 31.00 & \\
\hline & 3.61808100 & $-0.750100000 \mathrm{E}-02$ \\
\hline & 0.996656000 & 0.108098000 \\
\hline & 0.390735000 & 0.278540000 \\
\hline D & 21.00 & \\
\hline & 0.122770000 & 0.477318000 \\
\hline & $0.366550000 \mathrm{E}-01$ & 0.448183000 \\
\hline & & \\
\hline
\end{tabular}

\section{Cartesian Coordinates of Fully Optimized Structures}

\section{$5.1 \quad\left(\mathrm{C}_{5} \mathrm{H}_{6}\right)_{2} \mathrm{Ba}$}

$\begin{array}{ll}\mathrm{H} & 2.3486535818 \\ \mathrm{H} & 3.1698517889 \\ \mathrm{H} & -2.9953868222 \\ \mathrm{H} & -2.1774965928 \\ \mathrm{C} & 2.4402288565 \\ \mathrm{C} & 2.8702650257 \\ \mathrm{C} & -2.7786523535 \\ \mathrm{C} & -2.3508446511 \\ \mathrm{C} & 2.2507424175 \\ \mathrm{H} & 1.9865337726 \\ \mathrm{C} & 2.9461743099 \\ \mathrm{Ba} & 0.0001318168 \\ \mathrm{C} & -2.9784830349 \\ \mathrm{H} & 3.314579354 \\ \mathrm{C} & -2.2874692811 \\ \mathrm{C} & 2.5640248766 \\ \mathrm{H} & -3.3758350799 \\ \mathrm{H} & -2.0566988743 \\ \mathrm{C} & -2.6759801926 \\ \mathrm{H} & 2.5849659984 \\ \mathrm{H} & -2.8005211323\end{array}$

$5.2\left(\mathrm{C}_{9} \mathrm{H}_{9}\right)_{2} \mathrm{Ba}$

$\begin{array}{ll}\text { C } & 2.370485091 \\ \text { C } & 2.3771160696 \\ \text { C } & 2.3805350398 \\ \text { C } & 2.3793800154 \\ \text { C } & 2.3745900079 \\ \text { C } & 2.3679510208 \\ \text { C } & 2.3624520481 \\ \text { C } & 2.3608560769\end{array}$

$$
\begin{array}{ll}
-1.1897921952 & -1.9906983819 \\
1.3530177816 & -1.6789848782 \\
0.8031079526 & -2.0671214096 \\
-1.7090051112 & -1.5618864192 \\
-0.662917393 & -1.0516611015 \\
0.6813685275 & -0.8865021383 \\
0.3907786869 & -1.091508936 \\
-0.9376758354 & -0.8251485236 \\
-1.225142313 & 0.2396373155 \\
-2.250984346 & 0.4514141487 \\
0.9500987659 & 0.5069382793 \\
0.7007533641 & -0.0161541627 \\
1.0459048608 & 0.1537498586 \\
1.8613743636 & 0.9573308802 \\
-1.1044918802 & 0.5846343386 \\
-0.2286742269 & 1.2026922114 \\
2.0421551588 & 0.2894374192 \\
-2.0234679793 & 1.1039811939 \\
0.1210159263 & 1.1895492567 \\
-0.3684569175 & 2.2743174866 \\
0.2929269382 & 2.2494682234
\end{array}
$$

\footnotetext{
$-1.8080530251$

$-2.012799594$

$-1.275530056$

0.0587723461

1. 3656328813

2.0336370805

1. 7502625755

0.6480481912
}
0.9772013313
$-0.4136912086$
$-1.6107560704$
$-2.0537600728$
$-1.5354356436$
$-0.2984424314$
1. 0785332242
1. 9511701841




$\begin{array}{ll}\mathrm{C} & 2.3640630939 \\ \mathrm{H} & 2.3453421119 \\ \mathrm{H} & 2.3558400792 \\ \mathrm{H} & 2.3607370336 \\ \mathrm{H} & 2.3587279963 \\ \mathrm{H} & 2.3513289848 \\ \mathrm{H} & 2.3412610046 \\ \mathrm{H} & 2.3325560462 \\ \mathrm{H} & 2.3301910904 \\ \mathrm{H} & 2.3352861163 \\ \mathrm{Ba} & -0.0001019486 \\ \mathrm{C} & -2.3599379269 \\ \mathrm{C} & -2.3618819564 \\ \mathrm{C} & -2.3683659823 \\ \mathrm{C} & -2.3759309923 \\ \mathrm{C} & -2.3808859819 \\ \mathrm{C} & -2.3811549558 \\ \mathrm{C} & -2.3765289263 \\ \mathrm{C} & -2.3695169072 \\ \mathrm{C} & -2.3630579075 \\ \mathrm{H} & -2.3288049155 \\ \mathrm{H} & -2.3315319606 \\ \mathrm{H} & -2.3419380001 \\ \mathrm{H} & -2.3535620155 \\ \mathrm{H} & -2.3612649995 \\ \mathrm{H} & -2.3617179597 \\ \mathrm{H} & -2.3546359146 \\ \mathrm{H} & -2.3438448854 \\ \mathrm{H} & -2.3339518858\end{array}$

\section{$5.3\left(\mathrm{C}_{9} \mathrm{H}_{9}\right) \mathrm{Ba}\left(\mathrm{C}_{5} \mathrm{H}_{5}\right)$}

$\begin{array}{ll}\mathrm{C} & 2.0118289451 \\ \mathrm{C} & 1.9488617204 \\ \mathrm{C} & 1.8465579982 \\ \mathrm{C} & 1.7524157306 \\ \mathrm{C} & 1.7107767779 \\ \mathrm{C} & 1.7414076237 \\ \mathrm{C} & 1.8300352339 \\ \mathrm{C} & 1.93483852 \\ \mathrm{C} & 2.0064795999 \\ \mathrm{H} & 2.0749512911 \\ \mathrm{H} & 1.9782762246 \\ \mathrm{H} & 1.8212341853 \\ \mathrm{H} & 1.6763270492 \\ \mathrm{H} & 1.6118709114 \\ \mathrm{H} & 1.6588615801 \\ \mathrm{H} & 1.795313477 \\ \mathrm{H} & 1.956902961 \\ \mathrm{H} & 2.0671192556 \\ \mathrm{Ba} & -0.4820644026 \\ \mathrm{C} & -3.1778885405 \\ \mathrm{C} & -3.2086454336 \\ \mathrm{C} & -3.2346700523 \\ \mathrm{C} & -3.2201394832 \\ \mathrm{C} & -3.1855069193 \\ \mathrm{H} & -3.2171335387 \\ \mathrm{H} & -3.2778918807 \\ \mathrm{H} & -3.3300284376 \\ \mathrm{H} & -3.3011971664\end{array}$
$-0.7572322438$
1. 9111227827
1.4933697328
$-0.6327235512$
$-2.4626383667$
$-3.1398275419$
$-2.347561432$
$-0.4566966977$
1. 6481425546
2. 9821049036
2. 9208953838
$-0.0106345041$
1.8740995987
0.8922105553
$-0.5067008271$
$-1.6680188881$
$-2.0483371166$
$-1.4697277098$
$-0.202926057$
1. 1593453757
1. 9795780123
2. 8640964684
1. 363176441
$-0.7751747313$
$-2.5504418199$
$-3.1317973582$
$-2.2473794542$
$-0.3109083304$
1. 7715594671
3.02535914

$\begin{array}{ll}0.5850276442 & -1.9203221671 \\ 1.7159333536 & -1.0859757208 \\ 2.0444708848 & 0.2780709242 \\ 1.4169172831 & 1.5335384601 \\ 0.1269184769 & 2.0929717451 \\ -1.2218846793 & 1.6946989824 \\ -1.9984207861 & 0.5250954095 \\ -1.8393766302 & -0.8686716316 \\ -0.8190907632 & -1.8344992425 \\ 0.8936360402 & -2.9604665659 \\ 2.6220419253 & -1.6852673635 \\ 3.1240956037 & 0.3994161884 \\ 2.1648590077 & 2.3181646112 \\ 0.1932212168 & 3.1730761802 \\ -1.8681140783 & 2.5644226006 \\ -3.054950924 & 0.7770421611 \\ -2.811971178 & -1.3530389354 \\ -1.252557088 & -2.8292461955 \\ 0.0003866115 & -0.1207672079 \\ 0.4514513538 & 1.0716594856 \\ 1.2037407512 & -0.1335089542 \\ 0.2894237403 & -1.2213631652 \\ -1.0280125695 & -0.6880068659 \\ -0.927561663 & 0.7291062705 \\ 0.8561393224 & 2.0728178321 \\ 2.2796446477 & -0.2077214594 \\ 0.549422608 & -2.2660532467 \\ -1.943303424 & -1.256897047\end{array}$

S16 
1.6046806216

0.2485624703

0.6675548818

2. 4973790906

2. 2379621662

0.4232175019

$-1.0541555483$

1. 5788572676

0.8449604709

0.1306638444

$-1.5508665574$

1. 1822390313

2.8660498646

$-0.5604448406$

2.0372509215

$-0.9778768347$

1.811683333

1.0735168487

0.0287208391

$-1.1044152044$

$-2.0881828632$

$-0.0200564877$

0.5969163585

$-1.2004379574$

$-1.8037581763$

$-0.1905232869$

0.9857962437

1.1306239498

$-2.8581884879$

$-0.8163530431$

$-2.2649767612$

1. 5585499194

$-1.5708103463$

$-0.4153175587$

$-0.3543093213$

$-1.5430397527$

$-2.4892633736$

$-0.6597566447$

\section{$5.5\left[\left(\mathrm{C}_{5} \mathrm{H}_{5}\right)\right] \mathrm{Sr}\left(\mathrm{C}_{8} \mathrm{H}_{8}\right)$}

$\begin{array}{ll}\mathrm{H} & 4.8252193919 \\ \mathrm{H} & -4.794504455 \\ \mathrm{H} & -0.0226088874 \\ \mathrm{H} & -0.0032459301 \\ \mathrm{H} & -4.8264561789 \\ \mathrm{C} & -0.014198864 \\ \mathrm{H} & 4.8218501198 \\ \mathrm{C} & -0.0023147627 \\ \mathrm{C} & 4.7541030702 \\ \mathrm{C} & -4.7378853268 \\ \mathrm{H} & -0.0292911354 \\ \mathrm{C} & -4.7543851621 \\ \mathrm{H} & 0.0176474678 \\ \mathrm{C} & 4.7521523922 \\ \mathrm{H} & 4.8034092119 \\ \mathrm{C} & -0.0182600133\end{array}$

2.2306496412

2. 0270851981

2. 7677794924

2. 677145708

1. 6195302217

1. 7508018933

1.1317654413

1.6935719139

1.175601526

1. 0683290579

1.2412500633

0.8529293995

1. 0224226005

0.5949112442

0.2351119571

0.7867665986
0.466234239

$-1.0567189466$

$-1.0092758502$

1. 2401128684

1.6017383277

$-0.6351405462$

$-1.9890890286$

0.7852554535

0.2438837104

$-0.5615957106$

$-2.6636612332$

0.843597813

2. 7661707273

$-1.0538978468$

2.2699267257

$-1.6799853628$ 


\begin{tabular}{|c|c|}
\hline $\mathrm{C}$ & 0.0104859558 \\
\hline $\mathrm{C}$ & 4.7429994685 \\
\hline Sr & -2.2039356068 \\
\hline $\mathrm{C}$ & -4.7296161844 \\
\hline $\mathrm{H}$ & -4.7785625114 \\
\hline $\mathrm{Sr}$ & 2.2050601452 \\
\hline $\mathrm{C}$ & -4.7560927728 \\
\hline $\mathrm{C}$ & 4.7398999137 \\
\hline $\mathrm{C}$ & -0.0120406339 \\
\hline $\mathrm{C}$ & 4.7340902557 \\
\hline $\mathrm{C}$ & 0.0167007456 \\
\hline $\mathrm{H}$ & -4.8302945693 \\
\hline $\mathrm{H}$ & -0.0190807475 \\
\hline $\mathrm{C}$ & -4.7408569883 \\
\hline $\mathrm{H}$ & 4.7978654482 \\
\hline $\mathrm{H}$ & 0.0278089074 \\
\hline $\mathrm{C}$ & 0.0008290246 \\
\hline $\mathrm{C}$ & 0.0127132233 \\
\hline $\mathrm{H}$ & 4.7865377116 \\
\hline $\mathrm{H}$ & -4.8007164073 \\
\hline $\mathrm{H}$ & 0.0019520385 \\
\hline $\mathrm{H}$ & 0.0212850936 \\
\hline
\end{tabular}

$5.6\left[\left(\mathrm{C}_{5} \mathrm{H}_{5}\right)\right] \mathrm{Ba}\left(\mathrm{C}_{8} \mathrm{H}_{8}\right)$

$\begin{array}{lll}\mathrm{H} & 5.1672155791 & 1.4369075982 \\ \mathrm{H} & -5.2668973328 & 2.2186027609 \\ \mathrm{H} & 0.0686509668 & 2.7517613641 \\ \mathrm{H} & 0.1210750362 & 1.2062808336 \\ \mathrm{H} & -5.3162645395 & 0.1704676561 \\ \mathrm{C} & 0.041752982 & 1.7379571826 \\ \mathrm{H} & 5.2330224549 & 2.1351234885 \\ \mathrm{C} & 0.0742360225 & 0.7624303966 \\ \mathrm{C} & 5.1303464768 & 0.758254856 \\ \mathrm{C} & -5.181282879 & 1.170652857 \\ \mathrm{H} & -0.0248195002 & 2.6863283834 \\ \mathrm{C} & -5.2069491448 & 0.088454815 \\ \mathrm{H} & 0.1025599804 & -1.0440402558 \\ \mathrm{C} & 5.1643364994 & 1.1270369567 \\ \mathrm{H} & 5.179226087 & -1.2485243179 \\ \mathrm{C} & -0.0154857837 & 1.6965386466 \\ \mathrm{C} & 0.0628183236 & -0.6580099779 \\ \mathrm{C} & 5.1363426155 & -0.660519685 \\ \mathrm{Ba} & -2.4224674771 & 0.0235768434 \\ \mathrm{C} & -5.1244324347 & 0.6293194391 \\ \mathrm{H} & -5.1568507215 & 1.1941704519 \\ \mathrm{Ba} & 2.4222468919 & -0.0177896893 \\ \mathrm{C} & -5.1658160399 & -1.1214426353 \\ \mathrm{C} & 5.1913580975 & -0.0638062562 \\ \mathrm{C} & -0.0635686057 & 0.662930961 \\ \mathrm{C} & 5.1742281012 & -1.1687174098 \\ \mathrm{C} & 0.014603301 & -1.6916294963 \\ \mathrm{H} & -5.2367112174 & -2.119748085 \\ \mathrm{H} & -0.1035308519 & 1.0489114608 \\ \mathrm{C} & -5.1148659361 & -0.7871057778 \\ \mathrm{H} & 5.2856986023 & -0.1191074982 \\ \mathrm{H} & 0.0237445207 & -2.6813944067 \\ \mathrm{C} & -0.0747624832 & -0.757484487 \\ \mathrm{C} & -0.042405757 & -1.7329754478 \\ \mathrm{H} & 5.2527418524 & -2.2102308307 \\ \mathrm{H} & & \end{array}$

1. 7490864318

1. 19711968

0.0229709247

$-1.2006576663$

$-2.2659731004$

$-0.0114001604$

1.0730107982

$-0.9026278689$

$-1.7373796535$

0.4885579406

1. 6918547569

2. 035848751

$-2.7544247498$

$-0.1905202548$

$-1.7027021577$

2.6755078808

$-0.7735799975$

0.6469159179

0.9292656789

$-0.3546594628$

$-1.2283916812$

1.0209977996
1. 775172049
0.5203731214
1. 0537250376
2. 690149376
2. 2622451813
0.6684641948
$-0.8208585753$
1. 701297167
0.9348026972
0.2694031298
$-1.1950528912$
1.189919011
2. 7558162422
$-0.4368418665$
1. 9141582397
$-0.750899026$
1. 7426963668
1. 0081689807
0.0965963657
$-1.0429926497$
$-1.9639629141$
$-0.0792386763$
0.446054918
$-1.2113437781$
$-1.725114835$
$-0.3180762822$
0.7684903586
0.8544558942
$-2.7382196805$
$-0.9338073239$
$-2.2867819869$
1. 212682019
$-1.6835942556$
$-0.6508330079$
$-0.5963102125$ 


$\begin{array}{llll}\mathrm{H} & -5.1380547476 & -1.4866499537 & -1.7573516082 \\ \mathrm{H} & -0.121799203 & -1.2012831991 & -2.6724512599 \\ \mathrm{H} & -0.0695212447 & -2.7467566166 & -1.0361277195\end{array}$

\section{Complete list of authors for Gaussian 98 program for Ref. 81}

Frisch, M. J.; Trucks, G. W.; Schlegel, H. B.; Scuseria, G. E.; Robb, M. A.; Cheeseman, J.R.; Zakrzewski, V. G.; Montgomery, J. A.; Stratmann, R. E.; Burant, J. C.; Dapprich, S.; Millam, J. M.; Daniels, A. D.; Kudin, K. N.; Strain, M. C.; Farkas, O.; Tomasi, J.; Barone, V.; Cossi, M.; Cammi, R.; Mennucci, B.; Pomelli, C.; Adamo, C.; Clifford, S.; Ochterski, J.; Petersson, G. A.; Ayala, P. Y.; Cui, Q.; Morokuma, K.; Malick, D. K.; Rabuck, A. D.; Raghavachari, K.; Foresman, J. B.; Cioslowski, J.; Ortiz, J. V.; Stefanov, B. B.; Liu, G.; Liashenko, A.; Piskorz, P.; Komaromi, I.; Gomperts, R.; Martin, R. L.; Fox, D. J.; Keith, T.; Al-Laham, M. A.; Peng, C. Y.; Nanayakkara, A.; Gonzalez, C.; Challacombe, M.; Gill, P. M. W.; Johnson, B.; Chen, W.; Wong, M. W.; Andres, J. L.; Gonzalez, C.; Head-Gordon, M.; Replogle, E. S.; Pople, J. A. GAUSSIAN 98 (Revision A.5), Gaussian, Inc.: Pittsburgh PA, 1998. 